\title{
Consistent derivation of stress-based generalized models in elastodynamics: application to layerwise plate models
}

\author{
Paul Bouteiller ${ }^{\mathrm{a}}$, Jeremy Bleyer ${ }^{\mathrm{a}, *}$, Karam $\mathrm{Sab}^{\mathrm{a}}$ \\ ${ }^{a}$ Laboratoire Navier, Ecole des Ponts, Univ Gustave Eiffel, CNRS, Marne-la-vallée, France
}

\begin{abstract}
The derivation of some class of generalized mechanical models relies on a specific stress expansion without any a priori assumption on the corresponding kinematics. In such situations, their extension to a dynamic setting is not easy, especially when deriving the corresponding generalized inertias since the kinematics is unknown. This paper shows how consistent generalized inertias can be obtained through the use of a complementary energy principle in elastodynamics. The methodology is then applied to extend a stress-based layerwise plate model in the dynamic setting. This approach is finally validated through modal analysis of various anisotropic composite laminates.
\end{abstract}

Keywords: generalized continuum, stress-based model, elastodynamics, variational principle, multilayered plates, layerwise plate model

\section{Introduction}

Many generalized mechanical models often rely on a specific Ansatz on the local 3D displacement field to yield a generalized continuum formulation e.g. beam or plate models or even Cosserat or strain-gradient continuum models obtained from the up-scaling of a heterogeneous Cauchy continuum at the microscopic level. In such situations, extension to

\footnotetext{
${ }^{*}$ Correspondence to: J. Bleyer, Laboratoire Navier, 6-8 av Blaise Pascal, Cité Descartes, 77455 Champssur-Marne, France, Tel : +33 (0)1 64153743

Email addresses: paul.bouteiller@enpc.fr (Paul Bouteiller), jeremy.bleyer@enpc.fr (Jeremy Bleyer), karam.sab@enpc.fr (Karam Sab)
}

URL: https://sites.google.com/site/bleyerjeremy/ (Jeremy Bleyer) 
dynamics can be straightforward since the same Ansatz can be used for the velocity field, yielding a direct formulation of the generalized inertial terms.

However, other generalized mechanical models may also be built based on a specific Ansatz of the local 3D stress field instead of the displacement field. One can mention for instance the bending gradient theory for laminated plates [1-4], its more general version called generalized Reissner [5, 6], stress-gradient continua [7, 8]. In such cases, the expression of the local 3D displacement is generally unknown. The generalized model equations are then obtained by injecting the stress expansion into the complementary energy principle and the local equilibrium equations. The corresponding generalized degrees of freedom are then obtained by duality with respect to the model generalized equilibrium equations.

A question therefore arises when extending this procedure to a dynamic setting. How one must compute the generalized inertias associated with the generalized velocities from the local density distribution of the underlying material ? In some instances, the model generalized degrees of freedom may have a simple physical interpretation, allowing for a simple definition of the generalized inertias but this may not always be the case as it will be seen later. One therefore needs a systematic procedure to obtain a consistent definition of the elastodynamic version of such stress-based models.

As regards plate models for laminates, a huge amount of literature has been devoted to displacement-based approaches including more-or-less refined kinematics through the plate thickness. For instance, one can mention third-order shear deformation theory [9 11, higherorder shear deformation theories [12, 13], Murakami's zig-zag functions [14, Reddy's layerwise model [15], etc. A unified presentation can be found in [16]. As already mentioned, the extension of such displacement-based models to a dynamic setting generally poses no specific difficulty. However, other works advocated the use of stress-based layerwise plate models for an accurate description of stress fields through the plate thickness, the first one being Reissner's derivation for homogeneous isotropic plates [17]. Pagano [18], employing the Hellinger-Reissner mixed variational formulation, proposed a stress-based local layerwise model assuming a polynomial expansion of the stress per layer. Suffering from a certain operational heaviness, simplified models inspired by Pagano's work have been proposed e.g. 
the M4-2n+1 model [19, 20] or the more-refined "M4-5n" model [19, 21 23], later renamed LS1 to reflect that this Layerwise model is Stress-based with a first (1) order expansion of the membrane stress. Later, the SCLS1 model [24] built upon the ideas of the LS1 model to provide a rigorous statically compatible construction from the local 3D stress field, again assuming an affine expression of the membrane stress per layer.

For such models, there is no direct hypothesis on the 3D displacement form with respect to the thickness variable. It is therefore not clear how to extend such models in a dynamic setting. In [25], the extension of the LS1 model to dynamics has been somehow postulated from the classical Reissner-Mindlin inertias due to the simple mechanical interpretation of the LS1 model generalized displacements.

In this work, we propose to rely on a stress-based variational principle in elastodynamics, as initially proposed by Toupin [26, 27] and assume an appropriate hypothesis on the local 3D momentum field (rather than the displacement). Variational principles in elastodynamics will first be briefly reviewed in section 2. The proposed methodology will be described in section 3 , including a first illustrative example on a simple shear-lag model (section 3.1). It will then be applied on the SCLS1 laywerwise plate model in section 4. Validation will be performed in section 5 on modal analysis of laminated plates. Section 6 will conclude this work.

\section{A brief review of variational principles in elastodynamics}

We ignore body forces for simplicity and consider an elastic strain potential $\psi(\varepsilon)$ and its associated dual stress potential $\psi^{*}(\boldsymbol{\sigma})$. Displacements $\boldsymbol{u}=0$ are fixed on some part $\partial \Omega_{u}$ of the boundary and traction boundary conditions $\boldsymbol{\sigma} \cdot \boldsymbol{n}=\boldsymbol{t}$ are assumed on the remaining part $\partial \Omega_{T}$. Introducing the stress impulse $\boldsymbol{T}(t)=\boldsymbol{T}\left(t_{0}\right)+\int_{t_{0}}^{t} \boldsymbol{\sigma}(u)$ du, Toupin's complementary elastodynamic principle states [27, 28] that the following functional is stationary:

$$
\mathcal{A}[\mathcal{T}]=\int_{t_{0}}^{t_{1}} \int_{\Omega}\left(\psi^{*}(\dot{\mathcal{T}})-\frac{1}{2 \rho}\|\operatorname{div} \mathcal{T}\|^{2}\right) \mathrm{d} \Omega \mathrm{dt}
$$


for all $\mathcal{T}$ such that $\dot{\mathcal{T}} \cdot \boldsymbol{n}=\boldsymbol{t}$ on $\partial \Omega_{T}$

An equivalent two-field principle can be obtained by introducing the momentum density $\boldsymbol{p}$ as an independent unknown, i.e. $\boldsymbol{p}=\operatorname{div} \boldsymbol{\mathcal { T }}$. The complementary elastodynamic principle is therefore equivalent to the following functional stationarity:

$$
\mathcal{A}[\boldsymbol{\sigma}, \boldsymbol{p}]=\int_{t_{0}}^{t_{1}} \int_{\Omega}\left(\psi^{*}(\boldsymbol{\sigma})-\frac{1}{2 \rho}\|\boldsymbol{p}\|^{2}\right) \mathrm{d} \Omega \mathrm{dt}
$$

for all $\boldsymbol{\sigma}, \boldsymbol{p}$ such that:

$$
\begin{aligned}
& \operatorname{div} \boldsymbol{\sigma}=\dot{\boldsymbol{p}} \text { on } \Omega \\
& \boldsymbol{\sigma} \cdot \boldsymbol{n}=\boldsymbol{t} \text { on } \partial \Omega_{T}
\end{aligned}
$$

Note that this principle is the dual of Hamilton's elastodynamics principle [29] which involves the displacement $\boldsymbol{u}$ and velocity $\boldsymbol{v}$ fields and expresses the stationarity of the following functional:

$$
\mathcal{H}[\boldsymbol{u}, \boldsymbol{v}]=\int_{t_{0}}^{t_{1}} \int_{\Omega}\left(\frac{1}{2} \rho\|\boldsymbol{v}\|^{2}-\psi\left(\nabla^{s} \boldsymbol{u}\right)\right) \mathrm{d} \Omega \mathrm{dt}+\int_{t_{0}}^{t_{1}} \mathcal{W}_{\text {ext }}(\boldsymbol{u}) \mathrm{dt}
$$

such that $\boldsymbol{v}=\boldsymbol{u}, \boldsymbol{u}=0$ on $\partial \Omega_{u}$ and where $\mathcal{W}_{\text {ext }}$ denotes the work of external loads.

Finally, note also that (2a) can be transformed to a three-field principle by relaxing the equilibrium equation (2b) using an additional Lagrange multiplier which can be interpreted as the displacement $\boldsymbol{u}$. This mixed formulation is the dynamic counterpart of the static Hellinger-Reissner principle, allowing to choose independent discretizations of the stress, momentum and displacement fields.

\section{Derivation of elastodynamics stress-based models}

\subsection{Illustration on the shear lag model}

The shear lag model is often used to model the stress redistribution occurring from the interaction between two bonded elastic layers under longitudinal traction. Let us consider the bi-material of Figure 1 in plane-stress state in the $\left(x_{1}, x_{2}\right)$ plane consisting of one central 


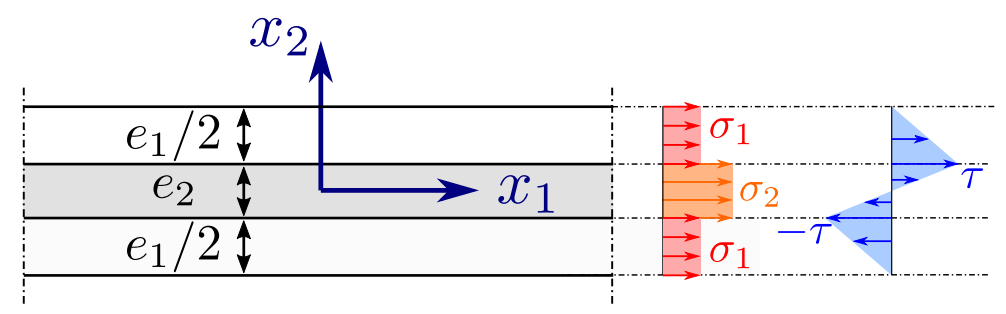

Figure 1: Geometry of the bi-material and shear-lag assumptions

core of thickness $e_{2}$ surrounded by two symmetric skins of thickness $e_{1} / 2$. Each material (skins and core) is isotropic elastic with Young modulus $E_{i}$ and shear modulus $\mu_{i}$ for $i=1,2$. For the sake of illustration, let us assume that the material density in both layers is described by a generic function $\rho\left(x_{2}\right)$. We consider a rectangular region $\Omega=\omega \times[-e / 2 ; e / 2]$ where $e=e_{1}+e_{2}$.

The shear-lag model can be obtained by assuming the following form for the local $2 \mathrm{D}$ stress-field $\boldsymbol{\sigma}\left(x_{1}, x_{2}\right)$ (see again Figure 1):

$$
\begin{aligned}
& \boldsymbol{\sigma}_{11}\left(x_{1}, x_{2}\right)= \begin{cases}\sigma_{1}\left(x_{1}\right) & \text { for }\left|x_{2}\right|>e_{2} / 2 \\
\sigma_{2}\left(x_{1}\right) & \text { for }\left|x_{2}\right|<e_{2} / 2\end{cases} \\
& \boldsymbol{\sigma}_{12}\left(x_{1}, x_{2}\right)= \begin{cases}\frac{2 \tau x_{2}}{e_{2}} & \text { for }\left|x_{2}\right|<e_{2} / 2 \\
\left( \pm e-2 x_{2}\right) \frac{\tau}{e_{1}} & \text { for } \pm x_{2} \geq e_{2} / 2\end{cases} \\
& \boldsymbol{\sigma}_{22}\left(x_{1}, x_{2}\right)=0
\end{aligned}
$$

The dynamic equation in the longitudinal direction can be written as:

$$
\dot{p}\left(x_{1}, x_{2}\right)= \begin{cases}\sigma_{1}^{\prime}\left(x_{1}\right)-\frac{2 \tau\left(x_{1}\right)}{e_{1}} & \text { for }\left|x_{2}\right|>e_{2} / 2 \\ \sigma_{2}^{\prime}\left(x_{1}\right)+\frac{2 \tau\left(x_{1}\right)}{e_{2}} & \text { for }\left|x_{2}\right|<e_{2} / 2\end{cases}
$$

A consistent use of the variational principle (2a) therefore requires to choose a piecewise- 
constant momentum with respect to $x_{2}$ :

$$
p\left(x_{1}, x_{2}\right)= \begin{cases}p_{1}\left(x_{1}\right) & \text { for }\left|x_{2}\right|>e_{2} / 2 \\ p_{2}\left(x_{1}\right) & \text { for }\left|x_{2}\right|<e_{2} / 2\end{cases}
$$

yielding the following generalized dynamic equations for $x_{1} \in \omega$ :

$$
\begin{aligned}
& \dot{p}_{1}\left(x_{1}\right)=\sigma_{1}^{\prime}\left(x_{1}\right)-\frac{2 \tau\left(x_{1}\right)}{e_{1}} \\
& \dot{p}_{2}\left(x_{1}\right)=\sigma_{2}^{\prime}\left(x_{1}\right)+\frac{2 \tau\left(x_{1}\right)}{e_{2}}
\end{aligned}
$$

Injecting expansions (4) and (6) into (2a) yields the following functional:

$$
\mathcal{A}\left[\sigma_{1}, \sigma_{2}, \tau, p_{1}, p_{2}\right]=\int_{t_{0}}^{t_{1}} \int_{\omega} \sum_{i=1}^{2}\left(\frac{e_{i}}{2 E_{i}} \sigma_{i}^{2}+\frac{e_{i}}{6 \mu_{i}} \tau^{2}-e_{i}\left\langle\frac{1}{2 \rho}\right\rangle_{i} p_{i}^{2}\right) \mathrm{dx} \mathrm{x}_{1} \mathrm{dt}
$$

which must be stationary for all $\sigma_{i}, \tau, p_{i}$ satisfying (7) and where $\langle\star\rangle_{1}$ (resp. $\left.\langle\star\rangle_{2}\right)$ denotes the average value over layer 1 (resp. 2).

Introducing $\lambda_{i}$ as the Lagrange multipliers associated with (7), the optimality conditions with respect to $\sigma_{i}$ and $\tau$ respectively yield:

$$
\begin{aligned}
e_{i} \frac{\sigma_{i}}{E_{i}}-\lambda_{i}^{\prime} & =0 \quad i=1,2 \\
\left(\frac{e_{1}}{3 \mu_{1}}+\frac{e_{2}}{3 \mu_{2}}\right) \tau+2\left(\frac{\lambda_{2}}{e_{2}}-\frac{\lambda_{1}}{e_{1}}\right) & =0
\end{aligned}
$$

whereas the optimality condition with respect to $p_{i}$ yields:

$$
-e_{i}\left\langle\frac{1}{\rho}\right\rangle_{i} p_{i}+\dot{\lambda}_{i}=0
$$

Defining $u_{i}=\lambda_{i} / e_{i}$, this quantity can be interpreted as the generalized displacement of layer $i$ with the following generalized constitutive laws and momentum/velocity relation:

$$
\begin{aligned}
\sigma_{i} & =E_{i} u_{i}^{\prime} \\
\tau & =\kappa\left(u_{1}-u_{2}\right) \\
p_{i} & =\left\langle\frac{1}{\rho}\right\rangle_{i}^{-1} \dot{u}_{i}
\end{aligned}
$$


where $\kappa=\frac{6}{\frac{e_{1}}{\mu_{1}}+\frac{e_{2}}{\mu_{2}}}$ is a shear interaction stiffness between both layers.

We can remark that this approach yields $m_{i}=1 /\langle 1 / \rho\rangle_{i}$ as the generalized inertia associated with the motion of layer $i$.

\subsection{General methodology}

We restrict here to the case of deriving a generalized continuum from a $3 \mathrm{D}$ continuum by dimension reduction along the $x_{3}$-coordinate, as it will be the case for the layerwise plate models investigated subsequently. For this purpose, we introduce $\Omega=\omega \times \mathcal{I}$ where $\omega$ is the plate reference plane and $\mathcal{I}$ the thickness interval along $x_{3}$. The methodology can however be directly extended to other situations such as the derivation of generalized beam models (reduction along $\left.x_{2}, x_{3}\right)$ for instance.

We assume that the local 3D stress $\boldsymbol{\sigma}(\boldsymbol{x})$ can be expressed linearly as a function of a vector of generalized stresses $\boldsymbol{\Sigma}\left(x_{1}, x_{2}\right)$ via some expansion along the $x_{3}$-direction. We further assume that this expansion leads to the following expression for the 3D stress divergence:

$$
\sigma_{i j, j}(\boldsymbol{x})=\sum_{k}\left(\boldsymbol{L}_{i}^{(k)} \boldsymbol{\Sigma}\left(x_{1}, x_{2}\right)\right) f_{i}^{(k)}\left(x_{3}\right) \quad \forall i
$$

where $\boldsymbol{L}_{i}^{(k)}$ are some differential operator with respect to $\left(x_{1}, x_{2}\right)$ and $f_{i}^{(k)}$ some shape functions of the $x_{3}$ variable forming an independent basis.

In static conditions, the local 3D equilibrium would translate into the following generalized equilibrium equations:

$$
\boldsymbol{L}_{i}^{(k)} \boldsymbol{\Sigma}\left(x_{1}, x_{2}\right)=0 \quad \forall i, k, \quad \forall\left(x_{1}, x_{2}\right) \in \omega
$$

As in the shear lag example, the Lagrange multipliers $U_{i}^{(k)}\left(x_{1}, x_{2}\right)$ associated with these equations can be interpreted as the generalized displacements of the model. They can be related to the local 3D displacements as follows:

$$
U_{i}^{(k)}\left(x_{1}, x_{2}\right)=\int_{\mathcal{I}} u_{i}(\boldsymbol{x}) f_{i}^{(k)}\left(x_{3}\right) \mathrm{d} \mathrm{x}_{3}
$$

Again, in stress-based models only an average relation between local and generalized displacements are known, not a full expansion. 
Following the same approach as in the previous section, we propose the extension to a dynamic setting by assuming a similar expansion as (11) for the momentum field $\boldsymbol{p}(\boldsymbol{x})$, namely:

$$
p_{i}(\boldsymbol{x})=\sum_{k} \boldsymbol{P}_{i}^{(k)}\left(x_{1}, x_{2}\right) f_{i}^{(k)}\left(x_{3}\right) \quad \forall i
$$

The generalized dynamic equations are therefore:

$$
\boldsymbol{L}_{i}^{(k)} \boldsymbol{\Sigma}\left(x_{1}, x_{2}\right)=\dot{\boldsymbol{P}}_{i}^{(k)}\left(x_{1}, x_{2}\right) \quad \forall i, k, \quad \forall\left(x_{1}, x_{2}\right) \in \omega
$$

and are strictly equivalent to the statisfaction of the local 3D dynamic equation.

Injecting the momentum expansion into (2a) yields:

$$
\begin{aligned}
\mathcal{A}[\boldsymbol{\Sigma}, \boldsymbol{P}] & =\int_{t_{0}}^{t_{1}} \int_{\omega}\left(\Psi^{*}(\boldsymbol{\Sigma})-\frac{1}{2} \boldsymbol{P}_{i}^{(k)} \int_{\mathcal{I}} \frac{1}{\rho} f_{i}^{(k)} f_{i}^{(j)} \mathrm{dx}_{3} \boldsymbol{P}_{i}^{(j)}\right) \mathrm{d} \omega \mathrm{dt} \\
& =\int_{t_{0}}^{t_{1}} \int_{\omega}\left(\Psi^{*}(\boldsymbol{\Sigma})-\frac{1}{2} \boldsymbol{P} \boldsymbol{D} \boldsymbol{P}\right) \mathrm{d} \omega \mathrm{dt}
\end{aligned}
$$

where $\Psi^{*}(\boldsymbol{\Sigma})=\int_{\mathcal{I}} \psi^{*}(\boldsymbol{\sigma}) \mathrm{dx}_{3}$ is the generalized stress energy density obtained from the local 3D stress expansion and in which we expressed the kinetic quadratic term in a condensed form involving the vector of generalized momenta $\boldsymbol{P}$ and a symmetric positive definite matrix $\boldsymbol{D}$. The elements of this matrix involve the computation of the products of the $f_{i}^{(k)}$ shape functions with the inverse density over $\mathcal{I}$.

Finally, let us recall that the stationarity conditions of $\mathcal{A}$ are constrained by the generalized dynamic equations (15), which we rewrite in the following condensed form:

$$
L \Sigma=\dot{P}
$$

The optimality conditions with respect to $\boldsymbol{P}$ therefore yield:

$$
\dot{U}=\boldsymbol{D P}
$$

linking the generalized momentum vector with the generalized velocity. The system generalized mass matrix is therefore given by $D^{-1}$. 


\section{Illustration on the Statically compatible layerwise model (SCLS1)}

To overcome the inaccurate estimations of local response near free-edges of equivalent single-layer theories, a collection of layerwise models including interlaminar stresses as unknown generalized forces have been developed in previous works [21, 22, 30]. In such works, the laminated plate can be seen as a superposition of Reissner-Mindlin-like plates coupled with each other through interlaminar forces. In the present paper, we consider a recent statically compatible version including first-order membrane stresses, called SCLS1, which ensures an accurate estimation of the shear stress and respect the free edge boundary conditions [24]. This stress-based model is derived from the Cauchy continuum 3D model by a single hypothesis: a polynomial (affine in the present case) expansion of the membrane stress per layer. The model construction is then obtained when enforcing the local 3D balance equations. In particular, the generalized kinematics are obtained by dualisation of the so-obtained generalized equilibrium equations. In particular, it makes no assumption whatsoever with respect to the underlying 3D kinematics. Therefore, there is no clear expression of the model inertial forces with respect to its generalized velocities. We will therefore apply the above described method to derive the generalized momentum-velocity relation.
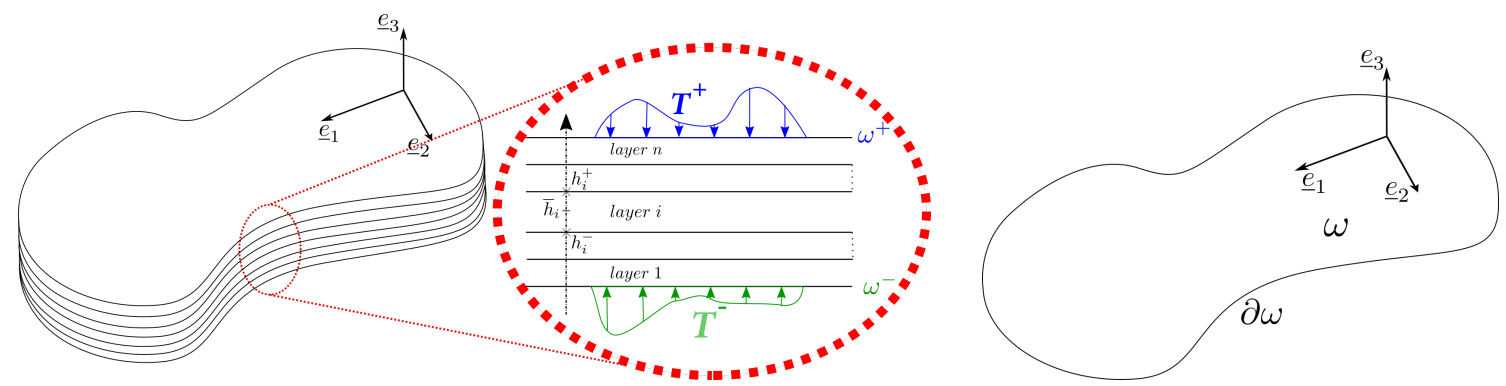

(a) Description of the $3 D$ laminated plate

(b) SCLS1-equivalent description of the $3 D$ laminated plate

Figure 2: Description of the laminated plate

\subsection{Problem description and notations}

We consider a multilayered plate, made of $n$ perfectly bonded layers, occupying the threedimensional domain $\Omega=\omega \times] h_{1}^{-} ; h_{n}^{+}[$where $\omega$ denotes the plate middle surface (Figure 2). 
Each layer is made of an orthotropic elastic material of compliance tensor $\mathbb{S}^{i}$. Let us fix the following notations:

- The lateral face is decomposed into two parts (respectively noted $\partial \Omega_{T}$ and $\partial \Omega_{D}$ ) where Neumann and Dirichlet boundaries conditions are applied.

- The plate is loaded on the upper $\omega^{+}$and lower $\omega^{-}$surface with a force distribution $\boldsymbol{T}^{+}=\left(T_{k}^{+}\right)$and $\boldsymbol{T}^{-}=\left(T_{k}^{-}\right)$respectively. Body forces are neglected.

- In the following, $x_{1}$ and $x_{2}$ refer to the in-plane coordinates while the $x_{3}$ coordinate refers to the out-of-plane component.

- The superscript $i$ and $j, j+1$ designate respectively the layer $i$ and the interface between the layer $j$ and $j+1$. By extension the superscript 0,1 designates the lower face $\omega^{-}$and the superscript $n, n+1$ the upper face $\omega^{+}$. Unless otherwise stated $i \in \llbracket 1 ; n \rrbracket, j \in \llbracket 1 ; n-1 \rrbracket$ and $k \in \llbracket 0 ; n \rrbracket$.

- $h_{i}^{-}, h_{i}^{+}$and $\overline{h_{i}}$ are, respectively, the bottom, top and mid-plane $x_{3}$-coordinate of layer $i$. Its thickness is $e^{i}=h_{i}^{+}-h_{i}^{-}$and $h_{j}^{+}=h_{j+1}^{-} \forall j \in \llbracket 1 ; n-1 \rrbracket$. By convention we also set $h_{0}^{+}=h_{1}^{-}$and $h_{n}^{+}=h_{n+1}^{-}$.

- Greek subscripts $\alpha, \beta, \gamma, \delta$ designate the in-plane components and take their values in $\llbracket 1 ; 2 \rrbracket$. Latin subscripts $i, j, k, l$ denote general components and take their values $\llbracket 1 ; 3 \rrbracket$.

- Einstein's summation convention is used.

\subsection{Stress field of the SCLS1 model}

The key idea of the SCLS1 model is to discretize the layered composite into a stack of plates in which the in-plane component $\sigma_{\alpha \beta}$ is a piecewise linear function. Originally derived in quasi-static conditions, we will retain the same hypothesis for the dynamic case. More precisely, we postulate that in each layer $i$, i.e. for $\left(x_{1}, x_{2}\right) \in \omega, x_{3} \in\left[h_{i}^{-}, h_{i}^{+}\right]$, the membrane stress field is written:

$$
\sigma_{\alpha \beta}^{i, 3 D}\left(x_{1}, x_{2}, x_{3}\right)=N_{\alpha \beta}^{i}\left(x_{1}, x_{2}\right) \frac{P_{0}^{i}\left(x_{3}\right)}{e^{i}}+M_{\alpha \beta}^{i}\left(x_{1}, x_{2}\right) \frac{12 P_{1}^{i}\left(x_{3}\right)}{e^{i^{2}}}
$$


where we make use of the polynomial basis $P_{k}^{i}\left(x_{3}\right):=L_{k}\left(\frac{x_{3}-\bar{h}_{i}}{e^{i}}\right)$ built from Legendre polynomials:

$$
L_{0}(y)=1 ; L_{1}(y)=y ; L_{2}(y)=-6 y^{2}+\frac{1}{2} ; L_{3}\left(x_{3}\right)=-2 y^{3}+\frac{3}{10} y
$$

Satisfaction of the local 3D balance equilibrium equations yields that $\sigma_{\alpha 3}^{i, 3 D}$ must be quadratic in $x_{3}$, whereas $\sigma_{33}^{i, 3 D}$ must be cubic in $x_{3}$. Taking into account traction continuity of $\sigma_{\alpha 3}^{i, 3 D}$ and $\sigma_{33}^{i, 3 D}$ at the interfaces, it can be easily established that such stress fields have the following expressions in layer $i$ :

$$
\begin{aligned}
\sigma_{\alpha 3}^{i, 3 D}\left(x_{1}, x_{2}, x_{3}\right) & =Q_{\alpha}^{i}\left(x_{1}, x_{2}\right) \frac{P_{0}^{i}\left(x_{3}\right)}{e^{i}}+\left[\left(\tau_{\alpha}^{i, i+1}-\tau_{\alpha}^{i-1, i}\right)\left(x_{1}, x_{2}\right)\right] P_{1}^{i}\left(x_{3}\right) \\
& +\left[Q_{\alpha}^{i}-\frac{e^{i}}{2}\left(\tau_{\alpha}^{i, i+1}+\tau_{\alpha}^{i-1, i}\right)\left(x_{1}, x_{2}\right)\right] \frac{P_{2}^{i}\left(x_{3}\right)}{e^{i}} \\
\sigma_{33}^{i, 3 D}\left(x_{1}, x_{2}, x_{3}\right) & =\left[\left(\frac{1}{2}\left(\nu^{i, i+1}+\nu^{i-1, i}\right)+\frac{e^{i}}{12}\left(\pi^{i,+}-\pi^{i,-}\right)\right)\left(x_{1}, x_{2}\right)\right] P_{0}^{i}\left(x_{3}\right) \\
& +\left[\left(\frac{e^{i}}{10}\left(\pi^{i,+}+\pi^{i,-}\right)+\frac{6}{5}\left(\nu^{i, i+1}-\nu^{i-1, i}\right)\right)\left(x_{1}, x_{2}\right)\right] P_{1}^{i}\left(x_{3}\right) \\
& +\left[\left(\frac{e^{i}}{12}\left(\pi^{i,+}-\pi^{i,-}\right)\left(x_{1}, x_{2}\right)\right] P_{2}^{i}\left(x_{3}\right)\right. \\
& +\left[\left(\frac{e^{i}}{2}\left(\pi^{i,+}+\pi^{i,-}\right)+\left(\nu^{i, i+1}-\nu^{i-1, i}\right)\right)\left(x_{1}, x_{2}\right)\right] P_{3}^{i}\left(x_{3}\right)
\end{aligned}
$$

where the in-plane fields appearing in (20), 222), 23) enjoy the following simple physical interpretations:

- $\boldsymbol{N}^{i}$ is the in-plane membrane force tensor in layer $i$

$$
N_{\alpha \beta}^{i}=\int_{h_{i}^{-}}^{h_{i}^{+}} \sigma_{\alpha \beta}^{i, 3 D}\left(x_{1}, x_{2}, x_{3}\right) \mathrm{d} x_{3}
$$

- $\boldsymbol{M}^{i}$ is the corresponding bending moment tensor

$$
M_{\alpha \beta}^{i}=\int_{h_{i}^{-}}^{h_{i}^{+}}\left(x_{3}-\bar{h}_{i}\right) \sigma_{\alpha \beta}^{i, 3 D}\left(x_{1}, x_{2}, x_{3}\right) \mathrm{d} x_{3}
$$

- $\boldsymbol{Q}^{i}$ is the resultant shear force vector

$$
Q_{\alpha}^{i}=\int_{h_{i}^{-}}^{h_{i}^{+}} \sigma_{\alpha 3}^{i, 3 D}\left(x_{1}, x_{2}, x_{3}\right) \mathrm{d} x_{3}
$$


- $\boldsymbol{\tau}^{i, i+1}$ is the interlaminar shear stress at interface $i, i+1$

$$
\sigma_{\alpha 3}^{i, 3 D}\left(x_{1}, x_{2}, h_{i}^{+}\right)=\sigma_{\alpha 3}^{i+1,3 D}\left(x_{1}, x_{2}, h_{i+1}^{-}\right)=\tau_{\alpha}^{i, i+1}\left(x_{1}, x_{2}\right)
$$

- $\nu^{i, i+1}$ is the interlaminar normal stress at interface $i, i+1$

$$
\sigma_{33}^{i, 3 D}\left(x_{1}, x_{2}, h_{i}^{+}\right)=\sigma_{33}^{i+1,3 D}\left(x_{1}, x_{2}, h_{i+1}^{-}\right)=\nu^{i, i+1}\left(x_{1}, x_{2}\right)
$$

As seen later, the generalized stress $\pi$ is related to the divergence of the interlaminar shear stress.

Finally, the traction boundary conditions on the top/bottom surfaces are given by:

$$
\begin{gathered}
\tau_{\alpha}^{0,1}=-T_{\alpha}^{-}, \nu^{0,1}=-T_{3}^{-} \\
\tau_{\alpha}^{n, n+1}=T_{\alpha}^{+}, \nu^{n, n+1}=T_{3}^{+}
\end{gathered}
$$

The generalized displacements are obtained by dualization of the model generalized balance equations. They can be related to weighted-averages of the three-dimensional displacement (see Appendix A.1.).

\subsection{Dynamic formulation of the model}

Inserting the stress field expression $(20-23)$ in the 3D equations of motion yields:

$$
\begin{aligned}
\dot{p}_{\alpha}\left(x_{1}, x_{2}, x_{3}\right)= & {\left[N_{\alpha \beta, \beta}^{i}+\left(\tau_{\alpha}^{i, i+1}-\tau_{\alpha}^{i-1, i}\right)\right] \frac{P_{0}^{i}\left(x_{3}\right)}{e^{i}} } \\
& +\left[M_{\alpha \beta, \beta}^{i}-Q_{\alpha}^{i}+\frac{e^{i}}{2}\left(\tau_{\alpha}^{i, i+1}+\tau_{\alpha}^{i-1, i}\right)\right] \frac{12 P_{1}^{i}\left(x_{3}\right)}{e^{i^{2}}} \\
\dot{p}_{3}\left(x_{1}, x_{2}, x_{3}\right)= & {\left[Q_{\alpha, \alpha}^{i}+\left(\nu^{i, i+1}-\nu^{i-1, i}\right)\right]\left[\frac{P_{0}^{i}\left(x_{3}\right)}{e^{i}}+\frac{P_{2}^{i}\left(x_{3}\right)}{e^{i}}\right] } \\
& +\left[\tau_{\alpha, \alpha}^{i, i+1}-\pi^{i,+}\right]\left[P_{1}^{i}\left(x_{3}\right)-\frac{P_{2}^{i}\left(x_{3}\right)}{2}\right]-\left[\tau_{\alpha, \alpha}^{i-1, i}-\pi^{i,-}\right]\left[P_{1}^{i}\left(x_{3}\right)+\frac{P_{2}^{i}\left(x_{3}\right)}{2}\right]
\end{aligned}
$$

where it can be seen that the stress divergence can be written in the form of (11). 
As a result, we postulate a piecewise linear (resp quadratic) interpolation of the in-plane (resp out-of-plane) momentum density in each layer:

$$
\begin{aligned}
\dot{p}_{\alpha}^{i}\left(x_{1}, x_{2}, x_{3}\right)= & \dot{r}_{\alpha}^{i, 0}\left(x_{1}, x_{2}\right) \frac{P_{0}^{i}\left(x_{3}\right)}{e^{i}}+\dot{r}_{\alpha}^{i, 1}\left(x_{1}, x_{2}\right) \frac{12 P_{1}^{i}\left(x_{3}\right)}{e^{i^{2}}} \\
\dot{p}_{3}^{i}\left(x_{1}, x_{2}, x_{3}\right)= & \dot{r}_{3}^{i, 0}\left(x_{1}, x_{2}\right)\left[\frac{P_{0}^{i}\left(x_{3}\right)}{e^{i}}+\frac{P_{2}^{i}\left(x_{3}\right)}{e^{i}}\right]+\dot{r}_{3}^{i,+}\left(x_{1}, x_{2}\right)\left[P_{1}^{i}\left(x_{3}\right)-\frac{P_{2}^{i}\left(x_{3}\right)}{2}\right] \\
& -\dot{r}_{3}^{i,-}\left(x_{1}, x_{2}\right)\left[P_{1}^{i}\left(x_{3}\right)+\frac{P_{2}^{i}\left(x_{3}\right)}{2}\right]
\end{aligned}
$$

In the static case [24], the equilibrium required that the generalized stress $\pi$ be continuous at the interfaces so that there was no need to distinguish $\pi^{i,+}, \pi^{i+1,-}$. In the dynamic setting, this continuity is no longer assured and, strictly speaking, we should keep both unknowns. However, to be consistent with the static case, we will assume $\dot{r}_{3}^{i,+}=\dot{r}_{3}^{i+1,-}=\dot{r}_{3}^{i, i+1}, \dot{r}_{3}^{i, i+1}$ now represent the momentum of the interface $i, i+1$. Such assumption ensure the continuity of $\pi$ when passing through the interface and we note $\pi^{i,+}=\pi^{i+1,-}=\pi^{i, i+1}$. Substituting (32) - 33 into (30) and writing the same system for the $i-1^{\text {th }}$ layer, we obtain $6 n+1$ equations of motion:

$$
\begin{aligned}
& N_{\alpha \beta, \beta}^{i}+\tau_{\alpha}^{i, i+1}-\tau_{\alpha}^{i-1, i}=\dot{r}_{\alpha}^{i, 0} \\
& M_{\alpha \beta, \beta}^{i}-Q_{\alpha}^{i}+\frac{e^{i}}{2}\left(\tau_{\alpha}^{i, i+1}+\tau_{\alpha}^{i-1, i}\right)=\dot{r}_{\alpha}^{i, 1} \\
& Q_{\alpha, \alpha}^{i}+\nu^{i, i+1}-\nu^{i-1, i}=\dot{r}_{3}^{i, 0} \\
& \tau_{\alpha, \alpha}^{k, k+1}-\pi^{k, k+1}=\dot{r}_{3}^{k, k+1}
\end{aligned}
$$

which correspond to the condensed form $\boldsymbol{L} \boldsymbol{\Sigma}=\dot{\boldsymbol{P}}$ introduced in 18$)$. Let us recall that the generalized stress $\boldsymbol{\Sigma}$ also has to fulfill the traction boundary conditions (29).

\subsection{Variational formulation}

Injecting the stress and momentum expansions, into the variational principle (2a) yields:

$$
\mathcal{A}[\boldsymbol{\Sigma}, \boldsymbol{P}, \boldsymbol{U}]=\int_{t_{0}}^{t_{1}} \int_{\omega}\left(\Psi^{*}(\boldsymbol{\Sigma})-\frac{1}{2} \boldsymbol{P} \boldsymbol{D} \boldsymbol{P}-(\boldsymbol{L} \boldsymbol{\Sigma}-\dot{\boldsymbol{P}}) \boldsymbol{U}\right) \mathrm{d} \omega \mathrm{dt}
$$

where the generalized displacement Lagrange multiplier $\boldsymbol{U}$ associated with the equation of motion as been included. The explicit expression of $\Psi^{*}$ and $\boldsymbol{D}$ is detailed in (A.2). 
* The optimality condition of the above functional with respect to the generalized displacement $\boldsymbol{U}$ leads to the dynamic equilibrium equations $34 \mathrm{a}-34 \mathrm{~d}$ ).

* The optimality condition with respect to the generalized stress $\Sigma$ leads to the generalized stress-strain constitutive laws (see [24] and Appendix A.2].

* The optimality condition with respect to the generalized momentum $\boldsymbol{P}$ leads to the generalized momentum-velocity relation:

$$
\dot{U}=D^{-1} \boldsymbol{P}
$$

\subsection{Special case of single-layer}

In this section, we consider a homogeneous plate consisting of a single anisotropic layer. The equations of the problem are obtained by particularizing the SCLS1 equations for $n=1$ : the dynamic equilibrium equations become:

$$
\begin{aligned}
& N_{\alpha \beta, \beta}+T_{\alpha}^{+}-T_{\alpha}^{-}=\dot{r}_{\alpha}^{0} \\
& M_{\alpha \beta, \beta}-Q_{\alpha}+\frac{e}{2}\left(T_{\alpha}^{+}+T_{\alpha}^{-}\right)=\dot{r}_{\alpha}^{1} \\
& Q_{\alpha, \alpha}+T_{3}^{+}-T_{3}^{-}=\dot{r}_{3}^{0} \\
& T_{\alpha, \alpha}^{+}-\pi^{+}=\dot{r}_{3}^{+} \\
& T_{\alpha, \alpha}^{-}-\pi^{-}=\dot{r}_{3}^{-}
\end{aligned}
$$

The first three equations are reduced in static (zero impulse) to the classical Reissner Mindlin plate equations. The stress field satisfies the Neumann-boundary conditions on the upper and lower faces of the plate. Under these assumptions (35) yield:

$$
\begin{aligned}
\mathcal{A}[\boldsymbol{\Sigma}, \boldsymbol{P}, \boldsymbol{U}]= & \int_{t_{1}}^{t_{2}} \int_{\omega}\left[w_{3 D}^{*}\left(\boldsymbol{N}, \boldsymbol{M}, \boldsymbol{Q}, \pi^{+}, \pi^{-}\right)-\int_{-\frac{e}{2}}^{\frac{e}{2}} \frac{1}{2 \rho} \boldsymbol{p} \cdot \boldsymbol{p} \mathrm{d} x_{3}\right] \mathrm{d} \omega \mathrm{dt} \\
& +\int_{t_{1}}^{t_{2}} \int_{\omega}\left[\left(N_{\alpha \beta, \beta}+T_{\alpha}^{+}-T_{\alpha}^{-}-\dot{r}_{\alpha}^{0}\right) U_{\alpha}+\left(M_{\alpha \beta, \beta}-Q_{\alpha}+\frac{e}{2}\left(T_{\alpha}^{+}+T_{\alpha}^{-}\right)-\dot{r}_{\alpha}^{1}\right) \Phi_{\alpha}\right] \mathrm{d} \omega \mathrm{dt} \\
& +\int_{t_{1}}^{t_{2}} \int_{\omega}\left[\left(Q_{\alpha, \alpha}+T_{3}^{+}-T_{3}^{-}-\dot{r}_{3}^{0}\right) U_{3}\right] \mathrm{d} \omega \mathrm{dt} \\
& +\int_{t_{1}}^{t_{2}} \int_{\omega}\left[\left(T_{\alpha, \alpha}^{+}-\pi^{+}-\dot{r}_{3}^{+}\right) V^{1,2}+\left(T_{\alpha, \alpha}^{-}-\pi^{-}-\dot{r}_{3}^{-}\right) V^{0,1}\right] \mathrm{d} \omega \mathrm{dt}
\end{aligned}
$$


with:

$$
\begin{aligned}
U_{\alpha}=\frac{1}{e} \int_{-\frac{e}{2}}^{\frac{e}{2}} u_{\alpha}\left(x_{1}, x_{2}, x_{3}, t\right) \mathrm{d} x_{3} & \Phi_{\alpha}=\int_{\frac{e}{2}}^{\frac{e}{2}} \frac{12 z}{e^{2}} u_{\alpha}\left(x_{1}, x_{2}, x_{3}\right) \mathrm{d} x_{3} \\
U_{3}=\int_{\frac{e}{2}}^{\frac{e}{2}}\left(\frac{1}{e}+\frac{P_{2}\left(x_{3}\right)}{e}\right) u_{3}\left(x_{1}, x_{2}, x_{3}\right) \mathrm{d} x_{3} & W_{ \pm}=\int_{\frac{e}{2}}^{\frac{e}{2}}\left(P_{1}(z) \pm \frac{P_{2}(z)}{2}\right) u_{3}\left(x_{1}, x_{2}, x_{3}, t\right) \mathrm{d} x_{3}
\end{aligned}
$$

* The optimality of (38) with respect to the generalized stress gives the generalized constitutive laws linking $\Sigma$ with the generalized deformation related to (39), see (A.13).

* The optimality with respect to the momentum density gives the generalized velocitiesmomentum density relation:

$$
\left(\begin{array}{c}
\dot{U}_{\alpha} \\
\dot{\Phi}_{\alpha} \\
\dot{U}_{3} \\
\dot{V}^{1,2} \\
\dot{V}^{0,1}
\end{array}\right)=\left(\begin{array}{ccccc}
\frac{1}{\rho e} & 0 & 0 & 0 & 0 \\
& \frac{12}{\rho e^{3}} & 0 & 0 & 0 \\
& & \frac{6}{5 \rho e} & -\frac{1}{10 \rho} & -\frac{1}{10 \rho} \\
& & & \frac{2 e}{15 \rho} & -\frac{e}{30 \rho} \\
\text { SYM } & & & & \frac{2 e}{15 \rho}
\end{array}\right)\left(\begin{array}{c}
r_{\alpha}^{0} \\
r_{\alpha}^{1} \\
r_{3}^{0} \\
r_{3}^{+} \\
r_{3}^{-}
\end{array}\right)
$$

First, it can be observed that the in-plane inertia and angular inertia (related to $\dot{U}_{\alpha}$ and $\dot{\Phi}_{\alpha}$ respectively) are given by the classical values $\rho e$ and $\rho e^{3} / 12$. However, the out-of-plane inertia is more complex since the SCLS1 model with one layer includes the additional degrees of freedom $W_{ \pm}$in addition to $U_{3}$. After inversion of the corresponding block, we obtain:

$$
\left(\begin{array}{c}
r_{3}^{0} \\
r_{3}^{+} \\
r_{3}^{-}
\end{array}\right)=\left(\begin{array}{ccc}
\rho e & \rho & \rho \\
\rho & \frac{9 \rho}{e} & \frac{3 \rho}{e} \\
\rho & \frac{3 \rho}{e} & \frac{9 \rho}{e}
\end{array}\right)\left(\begin{array}{c}
\dot{U}_{3} \\
\dot{V}^{1,2} \\
\dot{V}^{0,1}
\end{array}\right)
$$

We can note that the standard out-of-plane inertia $\rho e$ of a Reissner-Mindlin plate [25, 31] is recovered when zeroing the additional degrees of freedom $V^{j, j+1}=0$.

\section{Modal analysis of anisotropic multi-layered plates}

The strength of layerwise models lies in their ability to simulate highly anisotropic layered composites for a wide range of aspect ratios and stacking sequence. Their extension to the 
dynamic setting is here an additional step that we will validate by modal analysis tests on anisotropic plates. We are going to restrict ourselves to problems in which the boundaries are clamped. A $20 \times 20$ quadrilateral quadratic mesh is used to performs our simulations, see 24] for more details on the finite-element implementation. In the following, we will report the eigenfrequencies relative deviation $\left(\omega_{\text {ref }}-\omega_{F E M}\right) / \omega_{\text {ref }} \times$ from a given reference solution.

\section{1. (0,90) square plate laminate}

This example investigates the first 8 non-dimensional eigenfrequencies $\omega^{*}=\omega a^{2} \sqrt{\rho /\left(E_{T} h^{2}\right)}$ of a clamped square plate of side $a$ with an asymmetric $(0,90)$ stacking sequence of total thickness $h$. Our results will be compared to those found in [32] and a complete threedimensional model performed with Abaqus [33]. Figure 3 represents the deviation from the reference 3D solution for three different aspect ratios $a / h$. The corresponding tabulated results are given in Table A.2. We observe that the obtained results are better than those of [32] which relies on a conventional Galerkin approach and the first order shear deformation theory of Mindlin (FSDT).

\section{2. $(0,90)_{s}$ rectangular plate laminate}

Similarly, a rectangular plate of sides $a \times b$ and a symmetric $(0,90)_{s}=(0,90,90,0)$ stacking sequence is investigated. Results are again compared with a reference 3D FE solution and the results obtained in [34] using a p-Ritz method with the FSDT, see table A.3 for the tabulated results. Figure 4 again shows that the SCLS1 is much more accurate than the model from [34] for all mode numbers and aspect ratios.

\section{3. $(\theta,-\theta)_{n}$ square plate laminate}

Finally, a clamped square plate with a $(\theta,-\theta)_{n}$ lamination is now investigated for various orientations $\theta \in\{0,15,30,45\}$ and $n=1$ or $n=5$. Results are compared against those found in [32] and [35]. Figure 5 again shows the efficiency of the SCLS1 model compared to these references for all situations, except for the 10 layer case with $\theta= \pm 45^{\circ}$ where it is slightly less accurate. 


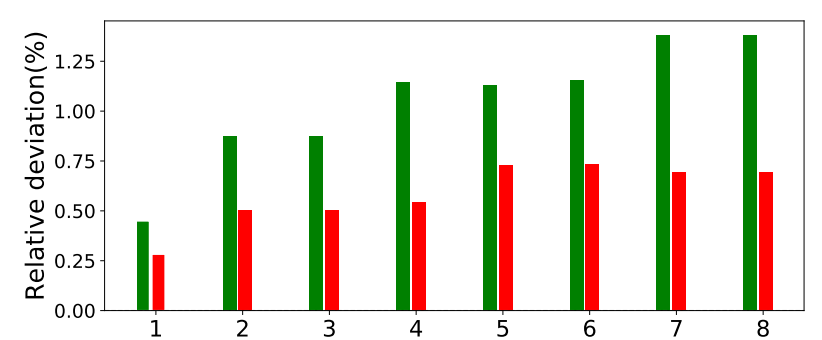

(a) $a / h=10$

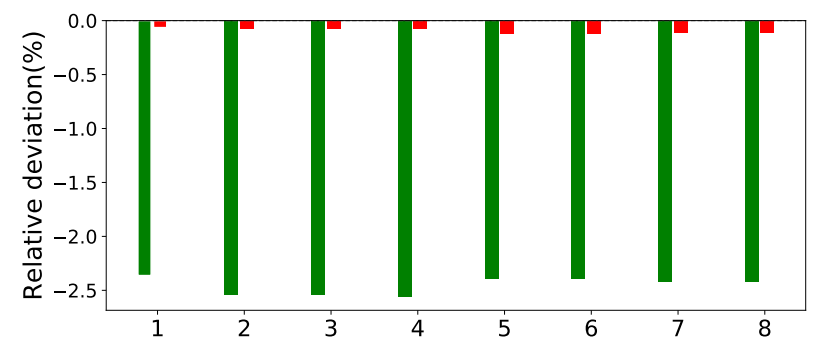

(c) $a / h=100$

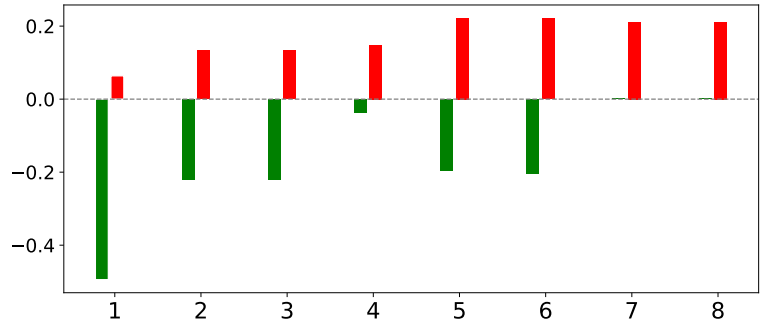

(b) $a / h=20$

Figure 3: Relative error compared to the three-dimensional model of the frequency parameter $\omega^{*}=$ $\omega a^{2} \sqrt{\rho /\left(E_{T} h^{2}\right)}$ for a clamped asymmetric plate $(0,90)$. The material properties used for the calculation are $E_{L} / E_{T}=25, G_{12}=G_{13}=0.5 E_{T}, G_{23}=0.2 E_{T}, \nu_{12}=0.25 . E_{L} / E_{T}=25, G_{12}=G_{13}=0.5 E_{T}$, $G_{23}=0.2 E_{T}, \nu_{12}=0.25$.

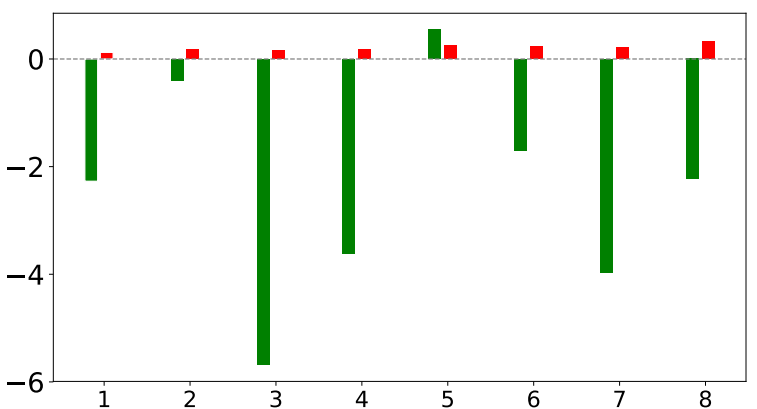

(a) $a / h=10$

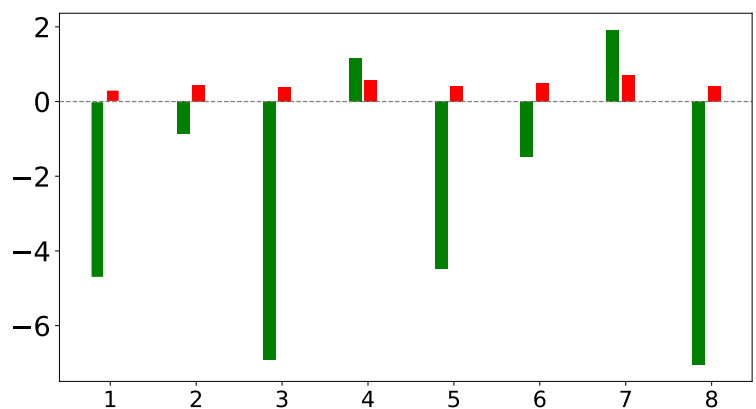

(b) $a / h=5$

Figure 4: Relative error with respect to the three-dimensional model of the frequency parameter $\bar{\omega}=$ $\left(\omega b^{2} / \pi^{2}\right) \sqrt{\left(\rho h / D_{0}\right)}\left(D_{0}=\frac{E_{T} h^{3}}{12\left(1-\nu_{21} \nu_{12}\right)}\right)$ for a symmetric $(0,90)_{s}$ laminate.

Overall, these three different comparisons show extremely convincing results since errors are less than $2 \%$ across the entire range of studies. This confirms the validity of our approach to determine the relevant inertia to be attributed to each of the SCLS1 model generalized 


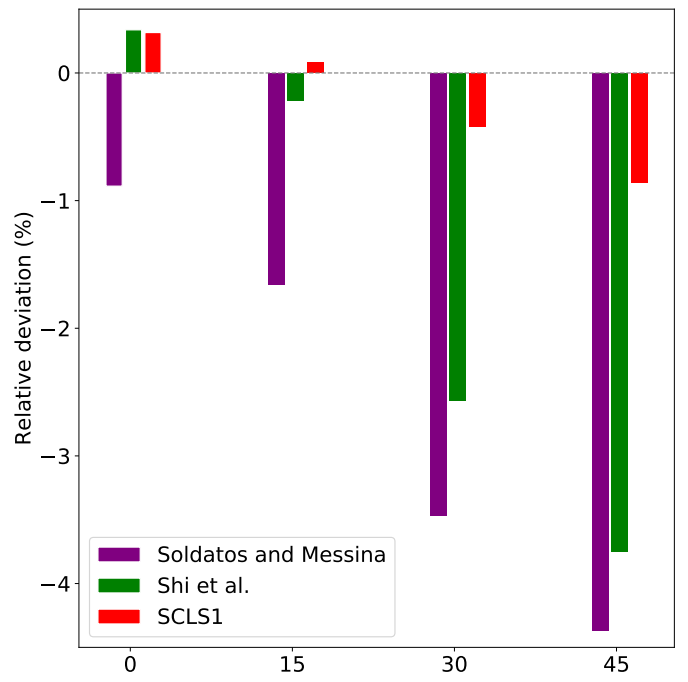

(a) $(\theta,-\theta)_{n}, n=1$

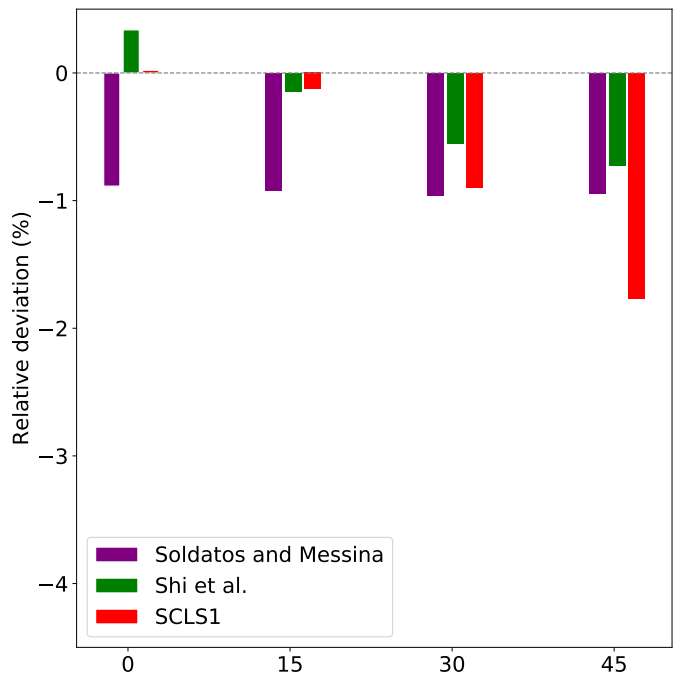

(b) $(\theta,-\theta)_{n}, n=5$

Figure 5: Relative error with respect to the three-dimensional model of the frequency parameter $\bar{\omega}=$ $\omega a^{2} \sqrt{\rho / E_{1} h^{2}}$ for a $(\theta,-\theta)_{n}$ laminated square plate; $a / h=20, E_{1} / E_{2}=40, G_{12}=G_{13}=G_{23}=0.5 E_{2}$, $\nu_{12}=0.25$.

displacements.

\subsection{Sandwich plate}

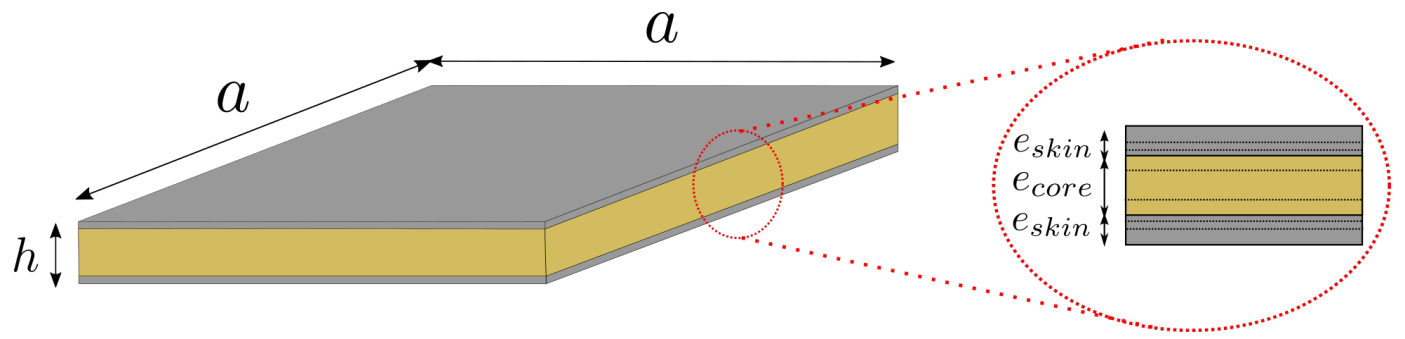

Figure 6: Geometry of the sandwich plate example, dashed lines represent the subdivision of a material layer into several "mathematical" layers

The aim of this section is to highlight the three-dimensional modeling capabilities of the SCLS1 model. For this purpose, we consider a square thick sandwich plate made of a flexible isotropic foam core, framed by two much stiffer isotropic steel skins (see Figure 6). We retain material [36] and geometric parameters [37] corresponding to a classical steel-polyurethane 
sandwich panel (see Table 1).

\begin{tabular}{l|lll}
\hline Material parameters & $E_{\text {skin }}=210 \times 10^{3} \mathrm{MPa}$ & $E_{\text {core }}=9.7 \mathrm{MPa}$ & $\nu_{\text {skin }}=\nu_{\text {core }}=0.33$ \\
& $\rho_{\text {skin }}=7.8 \times 10^{3} \mathrm{~kg} / \mathrm{m}^{3}$ & $\rho_{\text {core }}=64.2 \mathrm{~kg} / \mathrm{m}^{3}$ & \\
\hline Geometric parameters & $a=250 \mathrm{~mm}$ & $e_{\text {skin }}=2.41 \mathrm{~mm}$ & $e_{\text {core }}=54.1 \mathrm{~mm}$ \\
\hline
\end{tabular}

Table 1: Geometrical and material parameters for the sandwich plate example

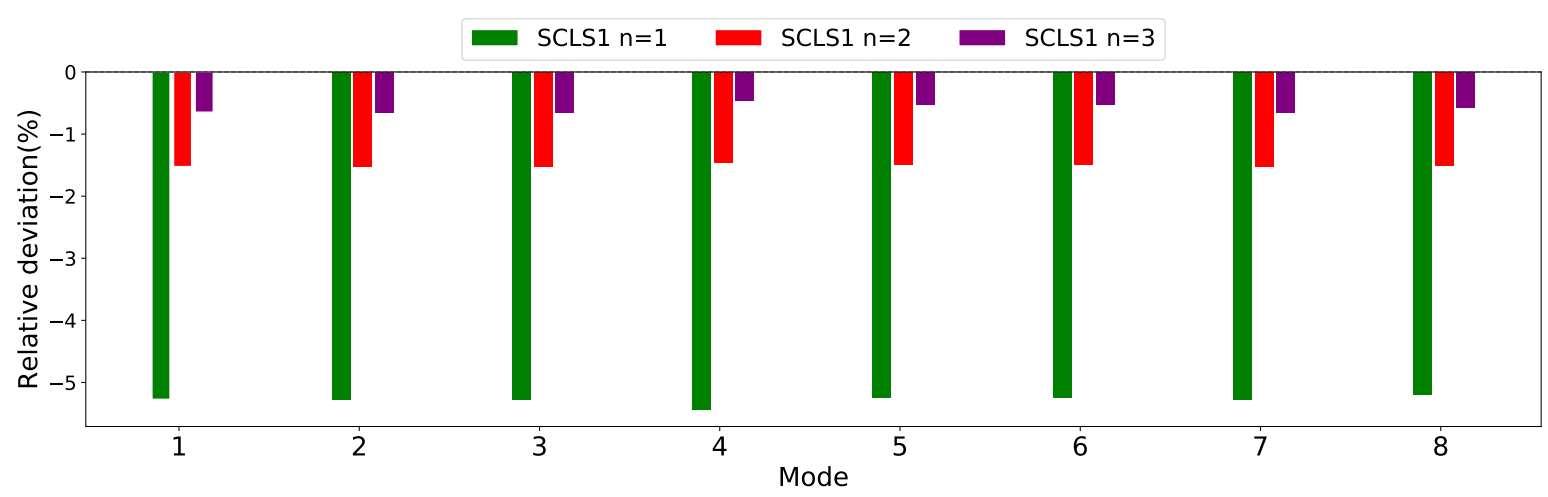

Figure 7: Relative error with respect to the three-dimensional model of the first 8 eigenfrequencies of the sandwich plate example

Inspecting the first 8 eigenmodes reveals that eigenmodes number 4, 5, 6 and 8 correspond to three-dimensional pinching and swelling modes which are perfectly captured by our model. Deformed configurations of the steel skins are represented in Figure 8.

The SCLS1 model can be viewed as an out-of-plane discretization of the three-dimensional continuum model when representing material layers by one or more "mathematical" layers (see Figure 6). It can be shown that the SCLS1 results converge towards the 3D solution when refining this layerwise discretization. This convergence is illustrated in Figure 7 where each ply (skins and core) is discretized by $n=1,2$ or 3 mathematical layers. A geometrical progression of the sub-layers thickness has been used to ensure a fast convergence as in [30]. It can be observed that using only one mathematical layer, although giving satisfying results 


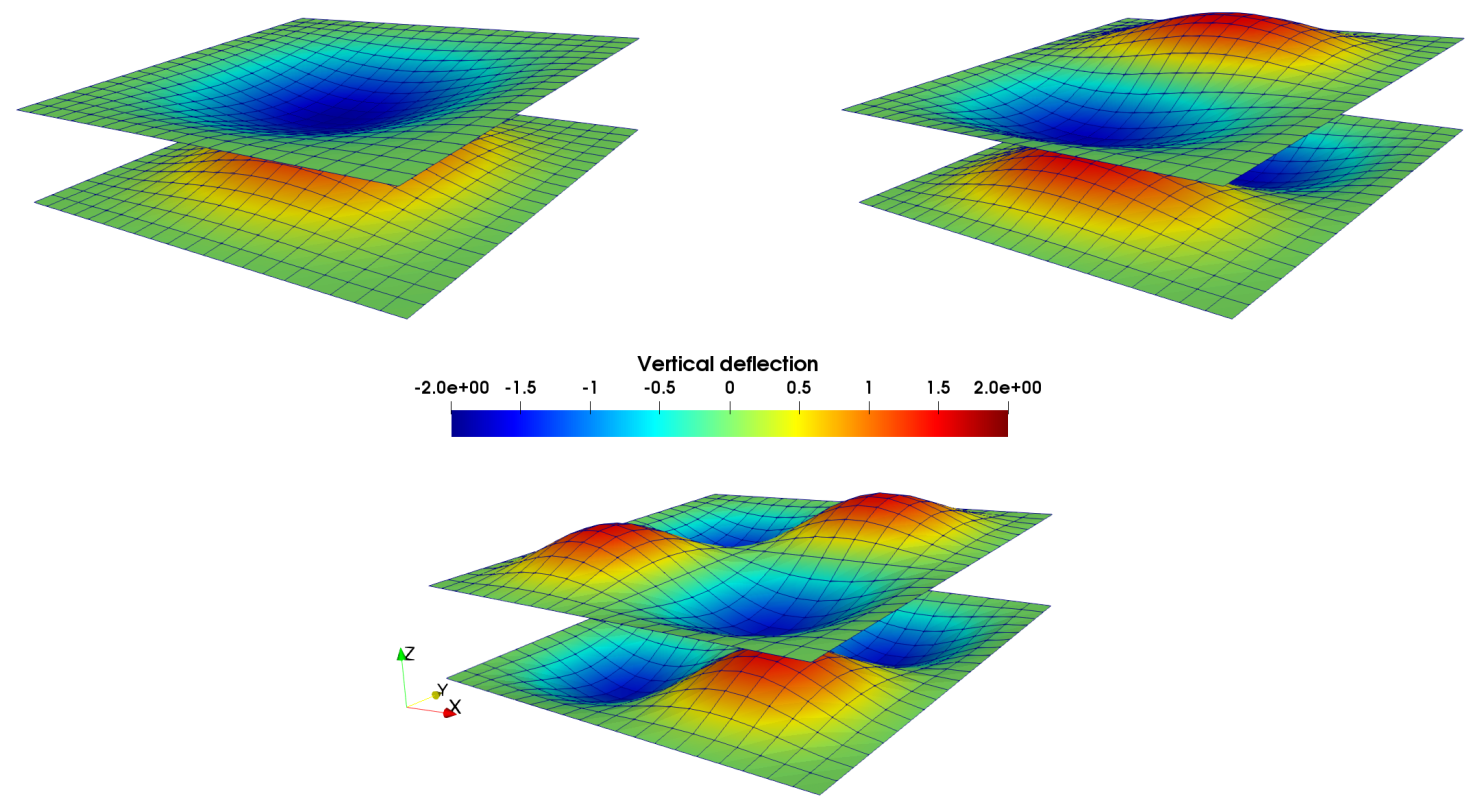

Figure 8: Representation of the $4,5,8^{\text {th }}$ mode

(relative error of 5\%), is not sufficient to ensure convergence even with an in-plane mesh refinement. This is most likely due to the strong stress variations inside the layers which are not well captured using only one mathematical layer. On the other hand, when increasing the number of mathematical layers, the stress field is better represented and the eigenfrequencies converge towards the 3D reference solution, as already previously mentioned in a quasi-static setting [24, 30].

\section{5. $(45,-45,45,-45)$ skew laminate plate}

We will conclude with a much more demanding modal analysis test. This example investigates the first 8 non-dimensional eigenfrequencies $\omega^{*}=\omega a^{2} \sqrt{\rho /\left(E_{T} h^{2}\right)}$ of a clamped rectangular plate with an asymmetric $(45,-45,45,-45)$ stacking sequence. The plate is skewed in its plane by an angle $\psi$. This configuration is visualized in Figure 9. Our results will be compared to those detailed in [38], the latter using the "B-spline RayleighRitz method (RRM)" and a Reissner Mindlin plate model (FOST). We will also mention 


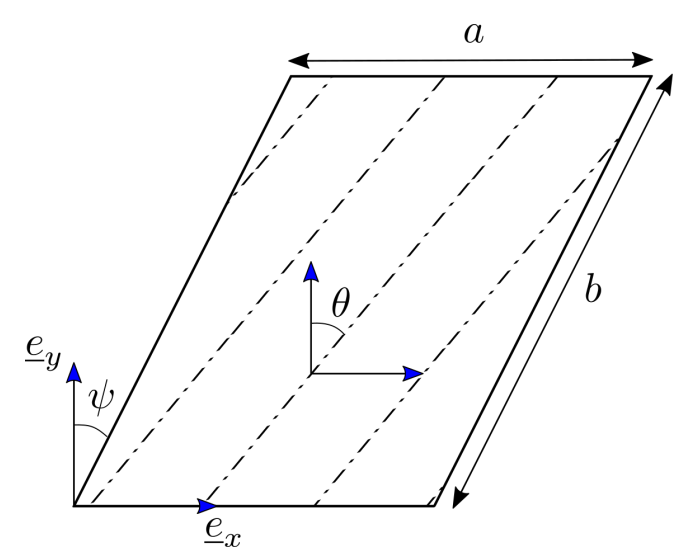

Figure 9: Geometry of the skew plate, in our numerical study $\theta=45^{\circ}, \psi \in\left\{0^{\circ}, 15^{\circ}, 30^{\circ}, 45^{\circ}\right\}$

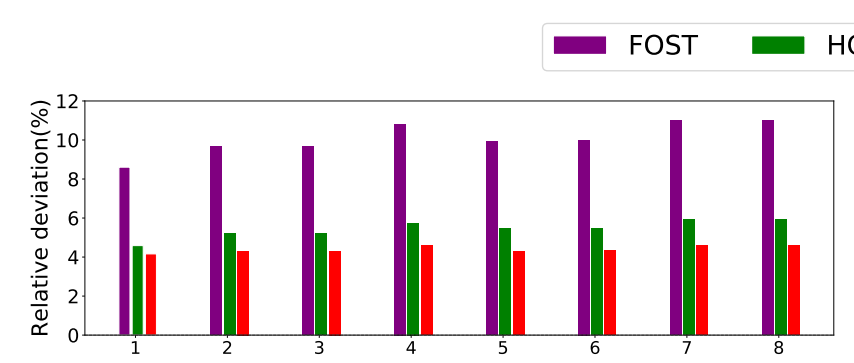

(a) $\psi=0^{\circ}$

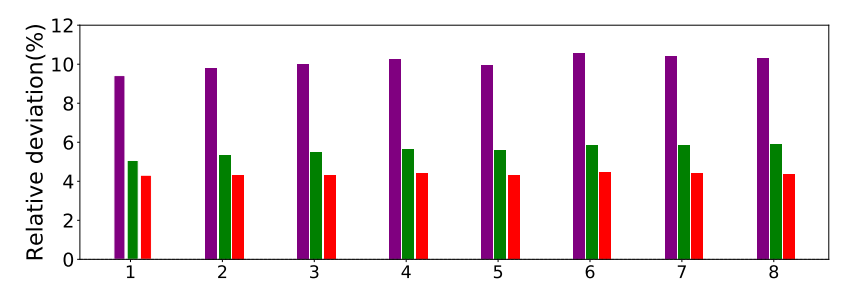

(c) $\psi=30^{\circ}$

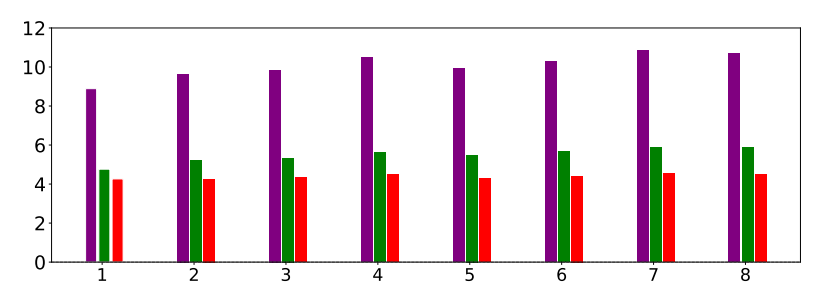

(b) $\psi=15^{\circ}$

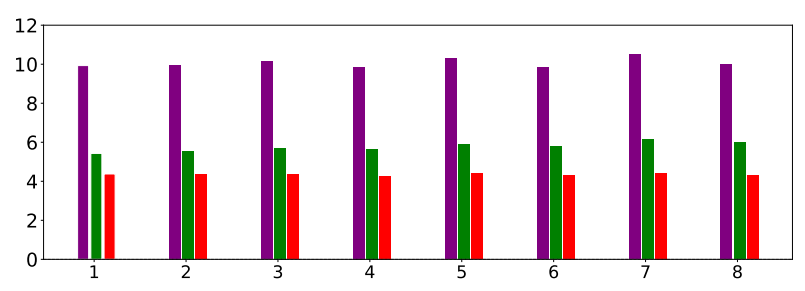

(d) $\psi=45^{\circ}$

Figure 10: Relative error with respect to the three-dimensional model of the frequency parameter $\lambda=$ $\omega a^{2} \sqrt{\rho / E_{2} h^{2}}$ for a skew laminate plate $(45 /-45 / 45 /-45) ; a / h=10, E_{1} / E_{2}=40, G_{12}=0.6 E_{2}, G_{13}=$ $G_{23}=0.5 E_{2}, \nu_{12}=\nu_{13}=\nu_{23}=0.25$

the results from a Higher Order Shear Theory (HOST) plate model found in [39]. Once again the comparison is visually illustrated on the Figure 10 , while the numerical results are recorded in Table A.6. This much more complex test strongly discriminates the coarser approach of 38 based on a first-order deformation theory. On the other hand, the HOST enriched model provides results of the same quality as our layerwise models. 


\section{Conclusion}

A general methodology to determine generalized inertias for models relying on a specific stress expansion has been presented. This principle has been used to identify non trivial generalized inertias of a stress-based layerwise model called SCLS1. Free vibration analysis of arbitrarily laminated plates used to assess our approach. Numerical simulations show a very good agreement with respect to reference solutions in a wide range of aspect ratios/anisotropic behaviors. The particular case of a single-layer was also investigated, the theoretical result differs from the usual Reissner-Mindlin kinematic approaches. The illustration of the SCLS1 model efficiency has been further illustrated on a sandwich plate example and the occurrence of pinching/swelling modes.

A natural extension of this work concerns its application to other stress-based generalized continuum models e.g. bending gradient or stress gradient models. Besides, for the sake of consistency, we adopted a pure stress-based approach in which local 3D equations of motion are satisfied exactly, yielding their generalized counterpart. However, a similar methodology can be applied in the case when such 3D equations of motion are satisfied only in a weak sense, yielding a mixed approach in which specific expansions are assumed for both stress and displacements. Finally, as regards the use of the SCLS1 model for the dynamic analysis of multi-layered plates, it appears that the obtained mass matrix is not diagonal, especially after inverting matrix $\boldsymbol{D}$ obtained from the proposed approach. Efficient dynamic computations (especially in the case of explicit time discretization schemes) often rely on the use of lumped mass matrices. For the present layerwise plate model, mass lumping must therefore be performed with respect to the generalized degrees of freedom to obtain a diagonal generalized mass matrix. However, there exist different mass lumping strategies which should be investigated in depth on such complex generalized continuum models.

\section{Acknowledgements}

The authors would like to thank Dassault Aviation for supporting this work. 


\section{Appendix A. Details of the SCLS1 model}

We refer to [24] for even more details on the construction of the SCLS1 model.

\section{Appendix A.1. Generalized displacement}

The generalized displacements of the SCLS1 model associated with each generalized equation of motion can be related to the 3D displacement $\boldsymbol{u}$ as follows:

$$
\begin{aligned}
& U_{\alpha}^{i}\left(x_{1}, x_{2}, t\right)=\int_{h_{i}^{-}}^{h_{i}^{+}} \frac{P_{0}^{i}\left(x_{3}\right)}{e^{i}} u_{\alpha}\left(x_{1}, x_{2}, x_{3}, t\right) \mathrm{d} x_{3} \\
& \Phi_{\alpha}^{i}\left(x_{1}, x_{2}, t\right)=\int_{h_{i}^{-}}^{h_{i}^{+}} \frac{12 P_{1}^{i}\left(x_{3}\right)}{e^{i^{2}}} u_{\alpha}\left(x_{1}, x_{2}, x_{3}, t\right) \mathrm{d} x_{3} \\
& U_{3}^{i}\left(x_{1}, x_{2}, t\right)=\int_{h_{i}^{-}}^{h_{i}^{+}}\left(\frac{P_{0}^{i}\left(x_{3}\right)}{e^{i}}+\frac{P_{2}^{i}\left(x_{3}\right)}{e^{i}}\right) u_{3}\left(x_{1}, x_{2}, x_{3}, t\right) \mathrm{d} x_{3} \\
& V^{j, j+1}\left(x_{1}, x_{2}, t\right)=W_{-}^{j}\left(x_{1}, x_{2}, t\right)-W_{+}^{j+1}\left(x_{1}, x_{2}, t\right) \\
& V^{n, n+1}\left(x_{1}, x_{2}, t\right)=W_{-}^{n}\left(x_{1}, x_{2}, t\right) \quad V^{0,1}\left(x_{1}, x_{2}, t\right)=-W_{+}^{1}\left(x_{1}, x_{2}, t\right) \\
& \text { where } W_{ \pm}^{i}\left(x_{1}, x_{2}, t\right)=\int_{h_{i}^{-}}^{h_{i}^{+}}\left(P_{1}^{i}\left(x_{3}\right) \pm \frac{P_{2}^{i}\left(x_{3}\right)}{2}\right) u_{3}\left(x_{1}, x_{2}, x_{3}, t\right) \mathrm{d} x_{3}
\end{aligned}
$$

where $U_{\alpha}^{i}\left(\operatorname{resp} U_{3}^{i}\right)$ correspond to the mean in-plane (resp out-of-plane) displacement of layer $i$. $\Phi_{\alpha}^{i}$ is the rotation of layer $i$. These generalized displacements are those of a ReissnerMindlin kinematics. $V^{j, j+1}$ is an additional out-of-plane degree of freedom related with the equation of motion involving $\pi^{j, j+1}$ and $\tau_{\alpha, \alpha}^{j, j+1}$ at the different interfaces.

\section{Appendix A.2. Constitutive laws}

Inserting the expression of the SCLS1 stress field and momentum density in (2a) and taking into account the generalized balance equations using their corresponding Lagrange 
multipliers (generalized displacements A.1) , the objective function becomes:

$$
\begin{aligned}
\mathcal{A}_{S C L S 1}= & \int_{t_{1}}^{t_{2}} \int_{\omega}\left[\sum_{i=1}^{n} \int_{h_{i}^{-}}^{h_{i}^{+}} \frac{1}{2} \boldsymbol{\sigma}_{3 D}^{i}: \mathbb{S}_{i}: \boldsymbol{\sigma}_{3 D}^{i} d x_{3}-\frac{1}{2 \rho^{i}} \boldsymbol{p}^{i} \cdot \boldsymbol{p}^{i} \mathrm{~d} x_{3}\right] \mathrm{d} \omega \mathrm{dt} \\
& +\int_{t_{1}}^{t_{2}} \int_{\omega}\left[\sum_{i=1}^{n}\left(N_{\alpha \beta, \beta}^{i}+\tau_{\alpha}^{i, i+1}-\tau_{\alpha}^{i-1, i}-\dot{r}_{\alpha}^{i, 0}\right) U_{\alpha}^{i}\right] \mathrm{d} \omega \mathrm{dt} \\
& +\int_{t_{1}}^{t_{2}} \int_{\omega}\left[\sum_{i=1}^{n}\left(Q_{\alpha, \alpha}^{i}+\nu^{i, i+1}-\nu^{i-1, i}-\dot{r}_{3}^{i, 0}\right) U_{3}^{i}\right] \mathrm{d} \omega \mathrm{dt} \\
& +\int_{t_{1}}^{t_{2}} \int_{\omega}\left[\sum_{i=1}^{n}\left(M_{\alpha \beta, \beta}^{i}-Q_{\alpha}^{i}+\frac{e^{i}}{2}\left(\tau_{\alpha}^{i, i+1}+\tau_{\alpha}^{i-1, i}\right)-\dot{r}_{\alpha}^{i, 1}\right) \Phi_{\alpha}^{i}\right] \mathrm{d} \omega \mathrm{dt} \\
& +\int_{t_{1}}^{t_{2}} \int_{\omega}\left[\sum_{k=0}^{n}\left[\tau_{\alpha, \alpha}^{k, k+1}-\pi^{k, k+1}-\dot{r}_{3}^{k, k+1}\right] V^{k, k+1}\right] \mathrm{d} \omega \mathrm{dt}
\end{aligned}
$$

which gives after integration by part:

$$
\begin{aligned}
\mathcal{A}_{S C L S 1}= & \int_{t_{1}}^{t_{2}} \int_{\omega}\left[w_{3 D}^{*}-\sum_{i=1}^{n} \int_{\omega} \boldsymbol{N}^{i}: \varepsilon^{i}+\boldsymbol{M}^{i}: \chi^{i}+\boldsymbol{Q}^{i} \cdot \gamma^{i} \mathrm{~d} \omega-\sum_{i=1}^{n} \int_{h_{i}^{-}}^{h_{i}^{+}} \frac{1}{2 \rho^{i}} \boldsymbol{p}^{i} \cdot \boldsymbol{p}^{i} \mathrm{~d} x_{3}\right] \mathrm{d} \omega \mathrm{dt} \\
& +\int_{t_{1}}^{t_{2}} \int_{\omega}\left[\sum_{j=1}^{n-1} \boldsymbol{\tau}^{j, j+1} \cdot \boldsymbol{D}_{\tau}^{j, j+1}+\nu^{i, i+1} D_{\nu}^{j, j+1}+\sum_{k=0}^{n} \pi^{k, k+1} \lambda^{k, k+1}\right] \mathrm{d} \omega \mathrm{dt} \\
& +\int_{t_{1}}^{t_{2}} \int_{\omega}\left[\sum_{i=1}^{n} r_{\alpha}^{i, 0} \dot{U}_{\alpha}^{i}+r_{3}^{i, 0} \dot{U}_{3}^{i}+r^{i, 1_{\alpha}} \dot{\Phi}_{\alpha}^{i}+r_{3}^{k, k+1} \dot{V}^{k, k+1}\right] \mathrm{d} \omega \mathrm{dt} \\
& +\int_{t_{1}}^{t_{2}} \int_{\omega} T_{\alpha}^{+}\left(U_{\alpha}^{n}+\frac{e^{n}}{2} \Phi_{\alpha}^{n}-V_{, \alpha}^{n, n+1}\right)-T_{3}^{+} U_{3}^{n}+T_{\alpha}^{-}\left(-U_{\alpha}^{1}+\frac{e^{1}}{2} \Phi_{\alpha}^{1}-V_{, \alpha}^{0,1}\right)-T_{3}^{-} U_{3}^{1} \mathrm{~d} \omega \mathrm{dt}
\end{aligned}
$$

where $w_{3 D}^{*}=\sum_{i=1}^{n} \int_{h_{i}^{-}}^{h_{i}^{+}} \frac{1}{2} \boldsymbol{\sigma}_{3 D}^{i}: \mathbb{S}_{i}: \boldsymbol{\sigma}_{3 D}^{i} d x_{3}$. The optimality of functional A.2 with respect to the generalized stresses yields the generalized constitutive laws which can be found in [24]. Here, we only provide the constitutive laws related to the $\pi^{0,1}$ and $\pi^{n, n+1}$ generalized stresses which were not present in the initial quasi-static model:

$$
\begin{aligned}
\lambda^{0,1}=\frac{\partial w_{3 D}^{*}}{\partial \pi^{0,1}}= & \frac{1}{105}\left(e^{1}\right)^{3} S_{3333}^{1} \pi^{0,1}-\frac{1}{140}\left(e^{1}\right)^{3} S_{3333}^{1} \pi^{1,2}-\frac{11}{210}\left(e^{1}\right)^{2} S_{3333}^{1} \nu^{0,1} \\
& -\frac{13}{420}\left(e^{1}\right)^{2} S_{3333}^{1} \nu^{1,2}-\frac{1}{12} e^{1} S_{\alpha \beta 33}^{1} N_{\alpha \beta}^{1}+\frac{1}{10} S_{\alpha \beta 33}^{1} M_{\alpha \beta}^{1} \\
\lambda^{n, n+1}=\frac{\partial w_{3 D}^{*}}{\partial \pi^{n, n+1}}=- & \frac{1}{140}\left(e^{n}\right)^{3} S_{3333}^{n} \pi^{n-1, n}+\frac{1}{105}\left(e^{n}\right)^{3} S_{3333}^{n} \pi^{n, n+1}+\frac{13}{420}\left(e^{n}\right)^{2} S_{3333}^{n} \nu^{n-1, n} \\
& +\frac{11}{210}\left(e^{n}\right)^{2} S_{3333}^{n} \nu^{n, n+1}+\frac{1}{12} e^{n} S_{\alpha \beta 33}^{n} N_{\alpha \beta}^{n}+\frac{1}{10} S_{\alpha \beta 33}^{n} M_{\alpha \beta}^{n}
\end{aligned}
$$


Appendix A.3. Momentum density-generalized displacement relation

The integral of the momentum density for layer $i$ is given by:

$$
\begin{aligned}
\int_{h_{i}^{-}}^{h_{i}^{+}} \boldsymbol{p}^{i} \cdot \boldsymbol{p}^{i} \mathrm{~d} x_{3}= & \int_{h_{i}^{-}}^{h_{i}^{+}}\left(r_{\alpha}^{i, 0} \frac{P_{0}^{i}\left(x_{3}\right)}{e^{i}}+r_{\alpha}^{i, 1} \frac{12 P_{1}^{i}\left(x_{3}\right)}{e^{i^{2}}}\right)^{2} \mathrm{~d} x_{3} \\
& +\int_{h_{i}^{-}}^{h_{i}^{+}}\left(r_{3}^{i, 0}\left[\frac{P_{0}^{i}\left(x_{3}\right)}{e^{i}}+\frac{P_{2}^{i}\left(x_{3}\right)}{e^{i}}\right]+r_{3}^{i, i+1}\left[P_{1}^{i}\left(x_{3}\right)-\frac{P_{2}^{i}\left(x_{3}\right)}{2}\right]\right. \\
& \left.+r_{3}^{i-1, i}\left[-P_{1}^{i}\left(x_{3}\right)-\frac{P_{2}^{i}\left(x_{3}\right)}{2}\right]\right)^{2} \mathrm{~d} x_{3} \\
= & \frac{1}{e^{i}}\left(r_{1}^{i, 0}\right)^{2}+\frac{12}{e^{i^{3}}}\left(r_{1}^{i, 1}\right)^{2}+\frac{1}{e^{i}}\left(r_{2}^{i, 0}\right)^{2}+\frac{12}{e^{i^{3}}}\left(r_{2}^{i, 1}\right)^{2}+\frac{6}{5 e^{i}}\left(r_{3}^{i, 0}\right)^{2}+\frac{2 e^{i}}{15}\left(r_{3}^{i, i+1}\right)^{2} \\
& +\frac{2 e^{i}}{15}\left(r_{3}^{i-1, i}\right)^{2}-\frac{1}{5} r_{1}^{i, 0}\left[r_{3}^{i-1, i}+r_{3}^{i, i+1}\right]-\frac{e^{i}}{15} r_{3}^{i-1, i} r_{3}^{i, i+1}
\end{aligned}
$$

Inserting A.5 in A.3 , the optimality condition with respect to the momentum density yields:

$$
\dot{\boldsymbol{U}}=\boldsymbol{D} \boldsymbol{R} \quad \Leftrightarrow \quad \boldsymbol{M} \dot{\boldsymbol{U}}=\boldsymbol{R} \quad \text { where } \boldsymbol{D}=\left(\begin{array}{c|c}
D_{u, \phi} & D_{U_{3}, r_{3}^{i, i+1}} \\
\hline D_{V, r_{3}^{i, 0}} & D_{V}
\end{array}\right)
$$

with:

$$
\begin{aligned}
& \boldsymbol{U}^{\top}=\left\{U_{1}^{1}, U_{2}^{1}, U_{3}^{1}, \Phi_{1}^{1}, \Phi_{2}^{1}, \ldots, U_{1}^{n}, U_{2}^{n}, U_{3}^{n}, \Phi_{1}^{n}, \Phi_{2}^{n}, V^{0,1}, \ldots, V^{n, n+1}\right\} \\
& \boldsymbol{R}^{\top}=\left\{r_{1}^{1,0}, r_{2}^{1,0}, r_{3}^{1,0}, r_{1}^{1,1}, r_{2}^{1,1}, \ldots, r_{1}^{n, 0}, r_{2}^{n, 0}, r_{3}^{n, 0}, r_{1}^{n, 1}, r_{2}^{n, 1}, r_{3}^{[0,1]}, \ldots, r_{3}^{[n, n+1]}\right\}
\end{aligned}
$$




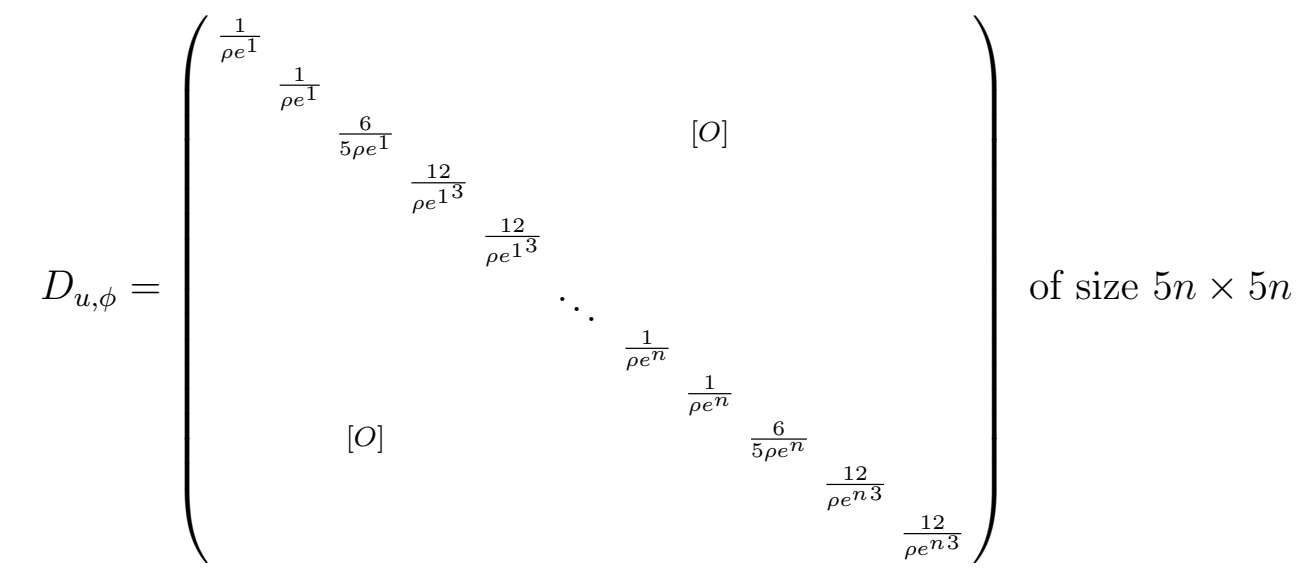

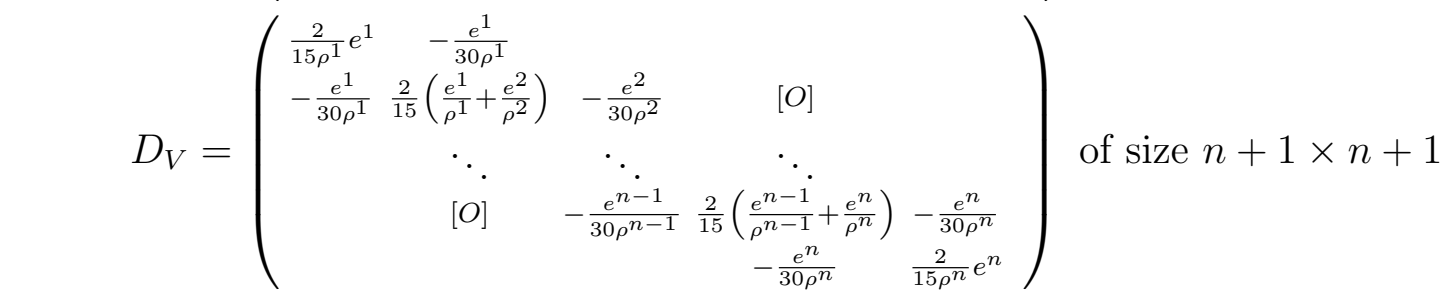

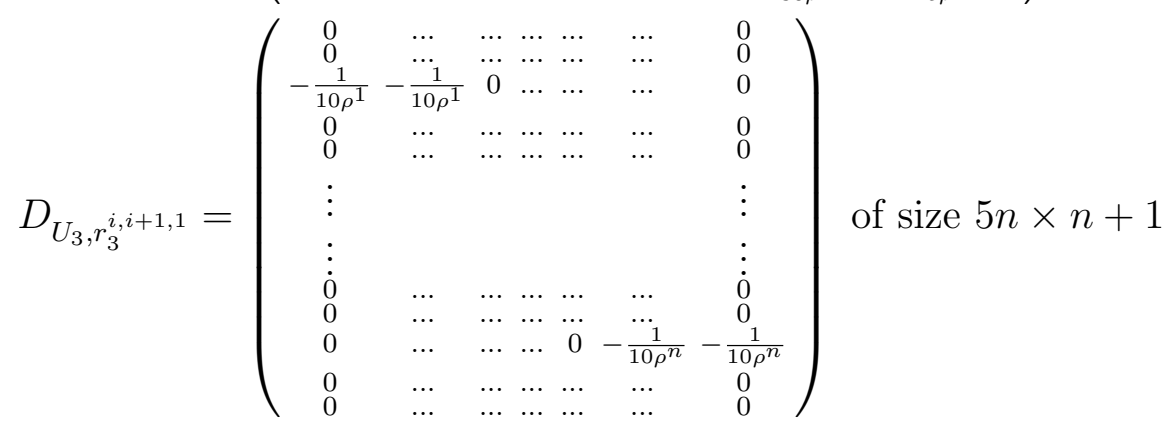

$$
\begin{aligned}
& D_{V, r_{3}^{i, 0}}=D_{U_{3}, r_{3}^{i, i+1,1}}^{\top}
\end{aligned}
$$


Appendix A.4. Constitutive laws for the single-layer plate

$$
\begin{aligned}
& \varepsilon_{\alpha \beta}=\frac{\partial w_{3 D}^{*}}{\partial N_{\alpha \beta}}=\frac{1}{e} S^{\alpha \beta \gamma \delta} N_{\alpha \beta}+S^{\alpha \beta 33}\left(\frac{1}{2}\left(T_{3}^{+}+T_{3}^{-}\right)+\frac{e}{12}\left(\pi^{+}-\pi^{-}\right)\right) \\
& \chi_{\alpha \beta}=\frac{\partial w_{3 D}^{*}}{\partial M_{\alpha \beta}}=\frac{12}{e^{3}} S^{\alpha \beta \gamma \delta} M_{\alpha \beta}+\frac{1}{e} S^{\alpha \beta 33}\left(\frac{6}{5}\left(T_{3}^{+}-T_{3}^{-}\right)+\frac{e}{10}\left(\pi^{+}+\pi^{-}\right)\right) \\
& \gamma_{\alpha}=\frac{\partial w_{3 D}^{*}}{\partial Q_{\alpha}}=\frac{24}{5 e} S^{\alpha 3 \gamma 3} Q_{\gamma}-\frac{2}{5} S^{\alpha 3 \gamma 3}\left(T_{\gamma}^{+}+T_{\gamma}^{-}\right) \\
& V^{0,1}=\frac{\partial w_{3 D}^{*}}{\partial \pi^{-}}=S^{3333}\left(\frac{e^{3}}{105} \pi^{-}-\frac{e^{3}}{140} \pi^{+}-\frac{11 e^{2}}{210} T_{3}^{-}-\frac{13 e^{2}}{420} T_{3}^{+}\right)-S^{\alpha \beta 33}\left(\frac{e}{12} N_{\alpha \beta}-\frac{1}{10} M_{\alpha \beta}\right) \\
& V^{1,2}=\frac{\partial w_{3 D}^{*}}{\partial \pi^{+}}=S^{3333}\left(\frac{e^{3}}{105} \pi^{+}-\frac{e^{3}}{140} \pi^{-}+\frac{13 e^{2}}{420} T_{3}^{-}+\frac{11 e^{2}}{210} T_{3}^{+}\right)+S^{\alpha \beta 33}\left(\frac{e}{12} N_{\alpha \beta}+\frac{1}{10} M_{\alpha \beta}\right)
\end{aligned}
$$

Appendix A.5. Tabulated results of modal analysis on laminated plates

Complete results of the investigated examples are available in Tables A.2, A.3, A.4, A.5.

\begin{tabular}{cccccccccc}
\hline$a / h$ & Source & \multicolumn{8}{c}{ Mode sequence number } \\
& & 1 & 2 & 3 & 4 & 5 & 6 & 7 & 8 \\
\hline \multirow{2}{*}{10} & 3D & 15.587 & 27.289 & 27.289 & 35.730 & 42.569 & 42.744 & 48.803 & 48.803 \\
& Shi et al. [32] & 15.517 & 27.050 & 27.050 & 35.320 & 42.087 & 42.249 & 48.128 & 48.128 \\
& SCLS1 & 15.544 & 27.153 & 27.153 & 35.538 & 42.262 & 42.435 & 48.467 & 48.467 \\
\hline \multirow{2}{*}{20} & 3D & 18.812 & 36.196 & 36.196 & 48.548 & 61.243 & 61.459 & 70.120 & 70.120 \\
& Shi et al. [32] & 18.905 & 36.276 & 36.276 & 48.566 & 61.363 & 61.584 & 70.119 & 70.119 \\
& SCLS1 & 18.802 & 36.153 & 36.153 & 48.481 & 61.115 & 61.332 & 69.980 & 69.980 \\
\hline \multirow{2}{*}{100} & 3D & 20.406 & 41.979 & 41.979 & 57.485 & 77.278 & 77.452 & 88.484 & 88.484 \\
& Shi et al. [32] & 20.888 & 43.048 & 43.048 & 58.955 & 79.129 & 79.308 & 90.624 & 90.624 \\
& SCLS1 & 20.418 & 41.997 & 41.997 & 57.509 & 77.317 & 78.392 & 88.527 & 88.527 \\
\hline
\end{tabular}

Table A.2: Frequency parameters $\omega^{*}=\omega a^{2} \sqrt{\rho /\left(E_{T} h^{2}\right)}$ for asymmetric cross-ply square plate 0/90. Material properties used in the calculation are $E_{L} / E_{T}=25, G_{12}=G_{13}=0.5 E_{T}, G_{23}=0.2 E_{T}, \nu_{12}=0.25$. 


\begin{tabular}{cccccccccc}
\hline$b / h$ & Source & 1 & 2 & 3 & 4 & 5 & 6 & 7 & 8 \\
\hline \multirow{2}{*}{0.001} & Liew [34] & 5.105 & 10.527 & 10.583 & 14.324 & 19.567 & 19.701 & 22.148 & 22.237 \\
& SCLS1 & 5.107 & 10.528 & 10.588 & 14.329 & 19.572 & 19.715 & 22.155 & 22.250 \\
\hline \multirow{2}{*}{0.100} & 3D & 4.049 & 6.591 & 7.905 & 9.550 & 10.021 & 12.236 & 13.138 & 13.812 \\
& Liew [34] & 4.141 & 6.617 & 8.354 & 9.895 & 9.967 & 12.443 & 13.659 & 14.120 \\
& SCLS1 & 4.045 & 6.579 & 7.894 & 9.534 & 10.000 & 12.209 & 13.112 & 13.769 \\
\hline \multirow{2}{*}{0.200} & 3D & 2.908 & 4.211 & 5.417 & 5.975 & 6.255 & 7.576 & 7.880 & 8.572 \\
& Liew [34] & 3.045 & 4.248 & 5.792 & 5.905 & 6.535 & 7.688 & 7.729 & 9.176 \\
& SCLS1 & 2.900 & 4.193 & 5.397 & 5.941 & 6.230 & 7.539 & 7.824 & 8.539 \\
\hline
\end{tabular}

Table A.3: Frequency parameters $\lambda=\left(\omega b^{2} / \pi^{2}\right) \sqrt{\left(\rho h / D_{0}\right)}$ for a clamped rectangular plate with $a / b=2$ and a symmetric $(0,90)_{s}$ layup. Reference $3 \mathrm{D}$ solution for the very thin case $b / h$ are not given.

\begin{tabular}{cccccc}
\hline \multirow{2}{*}{ Layup } & Source & \multicolumn{4}{c}{ Lamination angle $\theta$} \\
& & $0^{\circ}$ & $15^{\circ}$ & $30^{\circ}$ & $45^{\circ}$ \\
\hline$(\theta,-\theta)$ & 3D & 4.737 & 3.743 & 3.347 & 3.253 \\
& Soldatos and Messina [35] & 4.779 & 3.805 & 3.463 & 3.395 \\
& Shi et al. [32] & 4.721 & 3.751 & 3.433 & 3.375 \\
& SCLS1 & 4.722 & 3.740 & 3.361 & 3.281 \\
\hline$(\theta,-\theta)_{5}$ & 3D & 4.737 & 4.770 & 4.905 & 5.036 \\
& Soldatos and Messina [35] & 4.779 & 4.814 & 4.952 & 5.036 \\
& Shi et al. [32] & 4.721 & 4.776 & 4.932 & 5.025 \\
& SCLS1 & 4.736 & 4.777 & 4.949 & 5.077 \\
\hline
\end{tabular}

Table A.4: Frequency parameters $\bar{\omega}=\omega a^{2} \sqrt{\rho / E_{1} h^{2}} \omega^{*}=\omega a^{2} \sqrt{\rho /\left(E_{T} h^{2}\right)}$ for an asymmetric $(\theta,-\theta)_{n}$ square plate; $a / h=20, E_{1} / E_{2}=40, G_{12}=G_{13}=G_{23}=0.5 E_{2}, \nu_{12}=0.25$ 


\begin{tabular}{ccccccccc}
\hline Source & \multicolumn{9}{c}{ Mode sequence number: } & & & & \\
& 1 & 2 & 3 & 4 & 5 & 6 & 7 & 8 \\
\hline 3D & 12.673 & 24.078 & 24.078 & 27.255 & 32.635 & 32.635 & 34.478 & 40.166 \\
SCLS1 $n=1$ & 13.341 & 25.347 & 25.347 & 28.737 & 34.344 & 34.344 & 36.295 & 42.253 \\
SCLS1 $n=2$ & 12.866 & 24.445 & 24.445 & 27.653 & 33.119 & 33.119 & 35.005 & 40.771 \\
SCLS1 $n=3$ & 12.755 & 24.235 & 24.235 & 27.380 & 32.808 & 32.808 & 34.704 & 40.398 \\
\hline
\end{tabular}

Table A.5: 8 first eigenfrequencies $\omega / 2 \pi$ of the sandwich plate 


\begin{tabular}{|c|c|c|c|c|c|c|c|c|c|}
\hline \multirow{2}{*}{$\begin{array}{c}\text { Skew } \\
\text { angle } \psi\end{array}$} & \multirow{2}{*}{ Modèle } & \multicolumn{8}{|l|}{ Mode } \\
\hline & & 1 & 2 & 3 & 4 & 5 & 6 & 7 & 8 \\
\hline \multirow[t]{4}{*}{0} & $3 \mathrm{D}$ & 2.115 & 3.549 & 3.549 & 4.787 & 5.228 & 5.291 & 6.275 & 6.275 \\
\hline & FOST [38, 39] & 2.296 & 3.892 & 3.892 & 5.304 & 5.745 & 5.820 & 6.965 & 6.965 \\
\hline & HOST [39] & 2.212 & 3.734 & 3.734 & 5.062 & 5.513 & 5.581 & 6.647 & 6.647 \\
\hline & SCLS1 & 2.199 & 3.695 & 3.695 & 5.004 & 5.439 & 5.508 & 6.555 & 6.555 \\
\hline \multirow[t]{4}{*}{15} & $3 \mathrm{D}$ & 2.205 & 3.517 & 3.839 & 4.764 & 5.412 & 5.646 & 6.057 & 6.652 \\
\hline & FOST 39 & 2.401 & 3.856 & 4.217 & 5.264 & 5.949 & 6.227 & 6.714 & 7.363 \\
\hline & HOST [39] & 2.31 & 3.7 & 4.044 & 5.032 & 5.709 & 5.965 & 6.413 & 7.043 \\
\hline & SCLS1 & 2.299 & 3.667 & 4.005 & 4.977 & 5.644 & 5.895 & 6.333 & 6.951 \\
\hline \multirow[t]{4}{*}{30} & $3 \mathrm{D}$ & 2.506 & 3.754 & 4.467 & 4.933 & 6.021 & 6.137 & 6.591 & 7.289 \\
\hline & FOST 38, 39] & 2.742 & 4.122 & 4.913 & 5.44 & 6.618 & 6.784 & 7.275 & 8.043 \\
\hline & HOST [39] & 2.633 & 3.955 & 4.713 & 5.211 & 6.358 & 6.495 & 6.976 & 7.718 \\
\hline & SCLS1 & 2.614 & 3.916 & 4.659 & 5.15 & 6.280 & 6.411 & 6.883 & 7.608 \\
\hline \multirow[t]{4}{*}{45} & $3 \mathrm{D}$ & 3.132 & 4.385 & 5.526 & 5.682 & 6.684 & 7.305 & 7.794 & 8.479 \\
\hline & FOST [38], 39] & 3.443 & 4.822 & 6.085 & 6.241 & 7.372 & 8.024 & 8.623 & 9.332 \\
\hline & HOST [39] & 3.302 & 4.629 & 5.842 & 6.004 & 7.079 & 7.727 & 8.273 & 8.987 \\
\hline & SCLS1 & 3.269 & 4.576 & 5.766 & 5.925 & 6.978 & 7.620 & 8.140 & 8.843 \\
\hline
\end{tabular}

Table A.6: Frequency parameters $\bar{\omega}=\omega a^{2} \sqrt{\rho / E_{1} h^{2}}$ of a clamped rectangular plate with an asymmetric $(45,-45,45,-45)$ stacking sequence; $a / h=10, E_{1} / E_{2}=40, G_{12}=0.6 E_{2}, G_{13}=G_{23}=0.5 E_{2}, \nu_{12}=\nu_{13}=$ $\nu_{23}=0.25$ 


\section{References}

[1] A. Lebée, K. Sab, A bending-gradient model for thick plates. part i: Theory, International Journal of Solids and Structures 48 (2011) 2878-2888.

[2] A. Lebée, K. Sab, A bending-gradient model for thick plates, part ii: Closed-form solutions for cylindrical bending of laminates, International Journal of Solids and Structures 48 (2011) 2889-2901.

[3] A. Lebée, K. Sab, Homogenization of thick periodic plates: Application of the bending-gradient plate theory to a folded core sandwich panel, International Journal of Solids and Structures 49 (2012) 2778-2792.

[4] K. Sab, A. Lebée, Homogenization of heterogeneous thin and thick plates, John Wiley \& Sons, 2015.

[5] A. Lebée, K. Sab, On the generalization of Reissner plate theory to laminated plates, part i: Theory, Journal of Elasticity 126 (2017) 39-66.

[6] A. Lebée, K. Sab, On the generalization of Reissner plate theory to laminated plates, part ii: Comparison with the bending-gradient theory, Journal of Elasticity 126 (2017) 67-94.

[7] S. Forest, K. Sab, Stress gradient continuum theory, Mechanics Research Communications 40 (2012) $16-25$.

[8] G. Hütter, K. Sab, S. Forest, Kinematics and constitutive relations in the stress-gradient theory: interpretation by homogenization, International Journal of Solids and Structures 193 (2020) 90-97.

[9] M. Murthy, An improved transverse shear deformation theory for laminated antisotropic plates (1981).

[10] A. Bhimaraddi, L. Stevens, A higher order theory for free vibration of orthotropic, homogeneous, and laminated rectangular plates (1984).

[11] J. Reddy, A refined nonlinear theory of plates with transverse shear deformation, International Journal of solids and structures 20 (1984) 881-896.

[12] T. Kant, K. Swaminathan, Analytical solutions for free vibration of laminated composite and sandwich plates based on a higher-order refined theory, Composite structures 53 (2001) 73-85.

[13] R. K. Khare, T. Kant, A. K. Garg, Free vibration of composite and sandwich laminates with a higherorder facet shell element, Composite Structures 65 (2004) 405-418.

[14] E. Carrera, On the use of the Murakami's zig-zag function in the modeling of layered plates and shells, Computers \& Structures 82 (2004) 541-554.

[15] J. Reddy, A generalization of two-dimensional theories of laminated composite plates, Communications in applied numerical methods 3 (1987) 173-180.

[16] E. Carrera, Theories and finite elements for multilayered, anisotropic, composite plates and shells, Archives of Computational Methods in Engineering 9 (2002) 87-140.

[17] E. Reissner, On bending of elastic plates, Quarterly of Applied Mathematics 5 (1947) 55-68.

[18] N. Pagano, Stress fields in composite laminates, International Journal of Solids and Structures 14 
(1978) 385-400.

[19] A. Chabot, Analyse des efforts à l'interface entre les couches des matériaux composites à l'aide de modèles multiparticulaires de matériaux multicouches (M4), Ph.D. thesis, 1997.

[20] A. D. Diaz, J.-F. Caron, Interface plasticity and delamination onset prediction, Mechanics of Materials 38 (2006) 648-663.

[21] T. Naciri, A. Ehrlacher, A. Chabot, Interlaminar stress analysis with a new multiparticle modelization of multilayered materials (M4), Composites Science and Technology 58 (1998) 337-343.

[22] J.-F. Caron, A. D. Diaz, R. P. Carreira, A. Chabot, A. Ehrlacher, Multi-particle modelling for the prediction of delamination in multi-layered materials, Composites science and technology 66 (2006) 755-765.

[23] A. D. Diaz, S. Chataigner, J.-F. Caron, et al., A layerwise finite element for multilayers with imperfect interfaces, Composite structures 93 (2011) 3262-3271.

[24] R. Baroud, K. Sab, J.-F. Caron, F. Kaddah, A statically compatible layerwise stress model for the analysis of multilayered plates, International Journal of Solids and Structures 96 (2016) 11-24.

[25] V. A. Duong, Développement en dynamique d'un élément fini multicouche avec interfaces imparfaites, Ph.D. thesis, Université Paris Est, 2008.

[26] R. Toupin, A variational principle for the mesh-type analysis of a mechanical system, Journal of applied mechanics-transactions of the ASME 19 (1952) 151-152.

[27] B. Fraeijs de Veubeke, The dual principles of elastodynamics: finite element applications, Technical Report, LTAS, 1971.

[28] B. Tabarrok, Complementary variational principles in elastodynamics, Computers \& structures 19 (1984) 239-246.

[29] A. Bedford, Hamilton's principle in continuum mechanics, volume 139, Pitman advanced publishing program Boston, MA, 1985.

[30] N. Saeedi, K. Sab, J.-F. Caron, Delaminated multilayered plates under uniaxial extension. part ii: Efficient layerwise mesh strategy for the prediction of delamination onset, International Journal of Solids and Structures 49 (2012) 3727-3740.

[31] A. Belounar, S. Benmebarek, M. N. Houhou, L. Belounar, Static, free vibration, and buckling analysis of plates using strain-based Reissner-Mindlin elements, International Journal of Advanced Structural Engineering 11 (2019) 211-230.

[32] J. W. Shi, A. Nakatani, H. Kitagawa, Vibration analysis of fully clamped arbitrarily laminated plate, Composite Structures 63 (2004) 115-122.

[33] M. Smith, Abaqus/standard user's manual, version 6.9, Providence, RI: Simulia (2009).

[34] K. Liew, Solving the vibration of thick symmetric laminates by Reissner/Mindlin plate theory and the 
p-Ritz method, Journal of Sound and Vibration 198 (1996) 343-360.

[35] K. P. Soldatos, A. Messina, The influence of boundary conditions and transverse shear on the vibration of angle-ply laminated plates, circular cylinders and cylindrical panels, Computer methods in applied mechanics and engineering 190 (2001) 2385-2409.

[36] A. Fam, T. Sharaf, Flexural performance of sandwich panels comprising polyurethane core and GFRP skins and ribs of various configurations, Composite Structures 92 (2010) 2927-2935.

[37] H. Tuwair, M. Hopkins, J. Volz, M. A. ElGawady, M. Mohamed, K. Chandrashekhara, V. Birman, Evaluation of sandwich panels with various polyurethane foam-cores and ribs, Composites Part B: Engineering 79 (2015) 262-276.

[38] S. Wang, Free vibration analysis of skew fibre-reinforced composite laminates based on first-order shear deformation plate theory, Computers \& Structures 63 (1997) 525-538.

[39] A. K. Garg, R. K. Khare, T. Kant, Free vibration of skew fiber-reinforced composite and sandwich laminates using a shear deformable finite element model, Journal of Sandwich Structures \& Materials 8 (2006) 33-53. 